\title{
Light Scattering from Polymer Films Having an Optically Anisotropic Rod-Like Texture in Relation to a Model of a Random Assembly of Disordered Rods*
}

\author{
Takeji Hashimoto, Yukinobu Murakami, Norio Hayashi, ${ }^{* *}$ \\ and Hiromichi KAWAI ${ }^{* * *}$ \\ Department of Polymer Chemistry, Faculty of Engineering, \\ Kyoto University, Kyoto, Japan.
}

(Received September 19, 1973)

\begin{abstract}
Depolarized light-scattering intensity distributions from polymer films having a nonspherulitic but so called "rod-like" structure (or "fibrillar" structure) were investigated both theoretically and experimentally. Experimental scattered intensity distributions which were photometrically measured for poly(tetrafluoroethylene) and denatured collagen films were compared with theoretical intensity distributions based upon an assumption of a random assembly of optically anisotropic and homogeneous rods.

The results of the comparison show that the theoretical results deviate from the experimental both at small and large scattering angles. The effects of internal disorders of anisotropy and orientation of the optical axes of scattering elements constituting the rods and those of poly-dispersity in the size of the rods are theoretically investigated in order to account for the deviation at large scattering angles. The deviation at small angles may be attributed to an interrod interference effect which is neglected in the theory.
\end{abstract}

KEY WORDS Light Scattering / Nonspherulitic Crystalline Texture / Poly(tetrafluoroethylene) / Denatured Collagen / Films / Size Distribution / Effect of Disorders / Anisotropic Rods /

In the previous papers, depolarized light-scattering patterns from unoriented polymer films having a nonspherulitic (or prespherulitic) but fibrillar (or rod-like) crystalline texture were qualitatively explained in terms of a model based upon an assembly of optically anisotropic and homogeneous rods with finite length and infinitesimally thin width oriented randomly in twoor three-dimensional space ${ }^{1-3}$ or with finite length and width oriented in three-dimensional space. ${ }^{4-6}$ The scattering which was designated as "rodlike scattering" was found in crystalline polymer films of poly(tetrafluoroethylene) (PTFE) ${ }^{7,8}$ poly (monochloro-trifluoroethylene), ${ }^{8-10}$ cellulose de-

* Presented in part at the 21st Symposium on Polymer Chemistry, Osaka, Japan, November 2, 1972.

** Present Address: The Central Research Laboratories, Mitsubishi Chemical Industries, Inc., HisamotoKamoi-cho, Kawasaki, Japan.

*** To whom all correspondence should be addressed. rivaties, ${ }^{11,12}$ denatured collagen, ${ }^{2,3,13}$ poly(ethyleneterephthalate), ${ }^{14}$ and in blown films of polyethylene, ${ }^{15}$ and etc.

The characteristics of the rod-like scattering as compared with that of the scattering from a spherulitic crystalline superstructure ${ }^{16}$ were manifested in that the intensity of the rod-like scattering continuously decreases with increasing scattering angle, while that of the spherulitic scattering passes through maximum at a scattering angle reciprocally related to an average size of spherulite. ${ }^{16}$

In this paper a quantitative test of the rod model with infinitesimally thin width was performed for PTFE films and denatured collagen films by comparing the theoretical scattering patterns and intensity distributions with those experimentally measured-photographically and photometrically. Moreover, the effects of inhomogeneities of the rod-like structure and poly- 
dispersity in the size of the structure on the rodlike scattering were considered in order to account for the discrepancy between the theoretical and experimental results at large scattering angles.

\section{TEST SPECIMENS AND PHOTOGRAPHIC LIGHT-SCATTERING PATTERNS}

The theories of the rod-like scattering were tested for two types of PTFE films and three types of denatured collagen films. The PTFE films were prepared by annealing the dispersion. A thin layer of the dispersion was deposited on a microscope slide glass by dipping the glass into the dispersion. The layer was then heattreated at two different temperatures; for films designated as PTFE-L the dispersion was heated at $412^{\circ} \mathrm{C}$ for $2 \mathrm{hr}$ and then cooled down to room temperature at a natural rate, while for those designated as PTFE- $\mathrm{H}$ the dispersion was heated at $460^{\circ} \mathrm{C}$ for $2 \mathrm{hr}$ and cooled down to room temperature at a natural rate.

Three types of denatured collagen films designated as C-1, C-2 and C-3 specimens in this paper were chosen from the collagen films designated as " $R C$ " specimens in our previous paper. $^{2}$ The collagen films were prepared, as described in detail in the previous paper, by casting a dilute solution of solubilized collagens from fresh steer hide by proteolitic enzymes except for collagenese.

Some typical results of the microscopic observations are shown in Figure 1 to $4 . \quad$ It is seen from the observations that the internal structures of these test specimens are not typical of a spherulitic structure but rather composed of more or less randomly arranged optically anisotropic fibrillar or sheet-like textures. The texture of the PTFE-L specimens is negatively birefringent, indicating that the chain axes must be oriented nearly perpendicular to the needlelike texture (see Figure 1a). On the other hand, the orientation of the chain axes is not clear from the microscopic observation alone of the texture of the PTFE-H specimens (see Figure 1b). The problem can be clearly solved, however, by means of the light scattering method as will be discussed next.

The textures of the collagen films as observed by a polarizing microscope under crossed-nicols (Figure 2) were seen to be bright when they are located parallel to the polarization axes, indicating that the chain axes are oriented at nearly $45^{\circ}$ to the long direction of the textures.

The textures observed under a polarizing microscope have sizes of the right of order to scatter visible light, and are considered to be scattering entities in the following discussions.

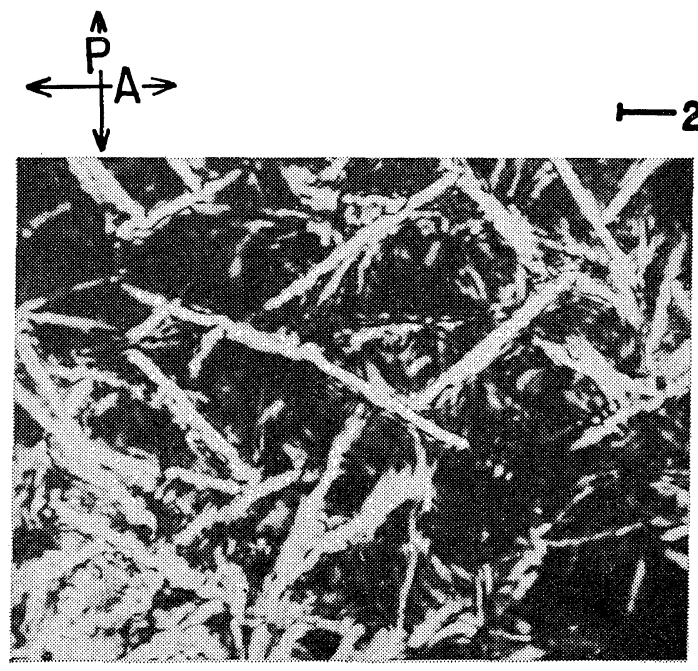

(a)
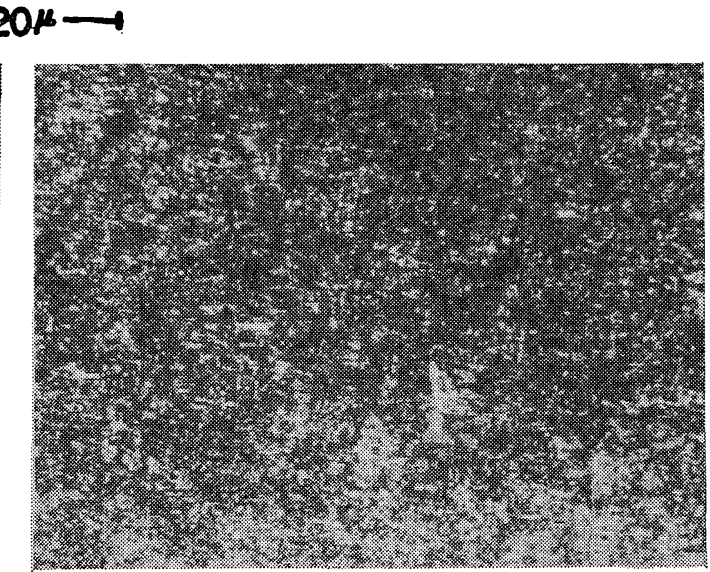

(b)

Figure 1. Polarizing micrographs of the PTFE-L (a) and PTFE-H (b) specimens (crossed-nicols). The polarizer and analyzer are set in vertical and horizontal directions. 


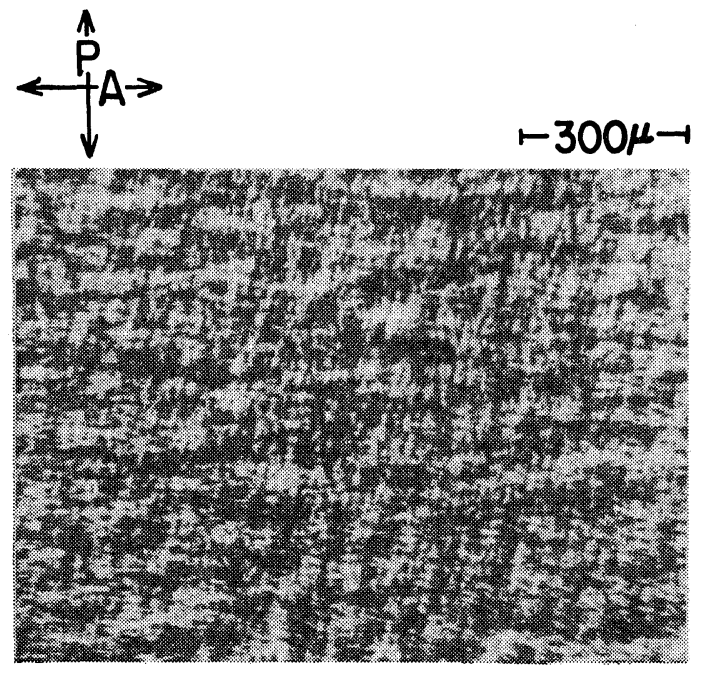

(a)

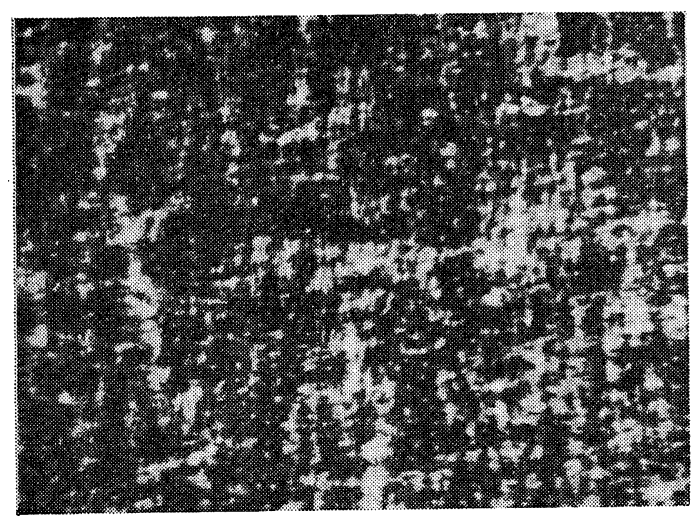

(b)

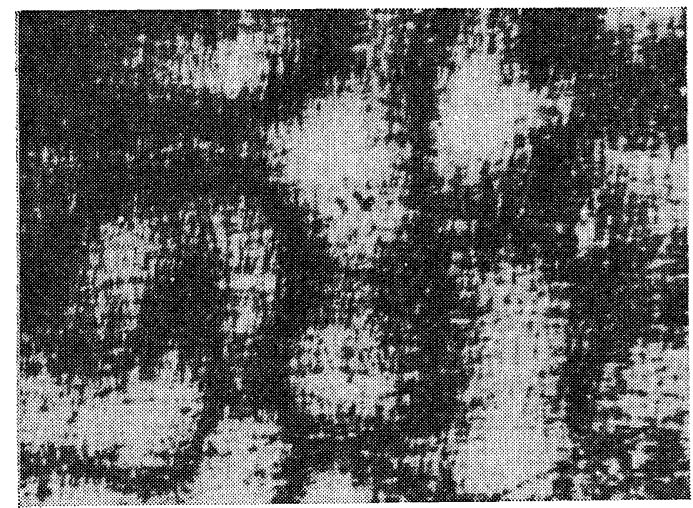

(c)

Figure 2. Polarizing micrographs of the denatured collagen films; (a) C-1, (b) C-2 and (c) C-3 specimens (crossed nicols). The symbols, $\mathbf{P}$ and $\mathbf{A}$ in the photograph show the directions of the polarizer and analyzer.
In the case of PTFE films, the textures may correspond to a "band" designated by Bunn, et al. ${ }^{17-19}$ or to a stack of the bands as shown in the scanning electron micrographs of the exterior surface of the specimens (Figures 3 and 4). The band may be considered to be a crystal lamella or to be a stack of the lamellae from previous microscopic observations, especially from the striations observed parallel to the band in the exterior surface of the specimens (the tendency which may be seen in Figures $3 b$ and 4b). It was thought that the chain axes were oriented perpendicular to the long direction of the band from the fine striations observed in fracture surfaces of specimens. ${ }^{17}$ Although the structure in the fracture surface was not examined in this study, the previous conclusion on the chain axis orientation seems to be valid at least for the PTFE-L specimens because of evidence that the band as observed by a polarizing microscope clearly showed negative birefringence (Figure 1a).

The textures of the PTFE-L specimens are considered to be oriented more or less randomly in a plane parallel to the film surface, since the thickness of the films prepared is about 10 to $20 \mathrm{~m} \mu$ which is smaller than the average size of the textures (about $70 \mathrm{~m} \mu$ ). The textures of the denatured collagen films are also considered to be oriented more or less randomly in a plane parallel to the film surface from X-ray photographs taken by irradiating X-ray parallel (b) and normal (a) to the film surface as seen, for example, in Figure 5. The photographs suggest a planar orientation of tropocollagen molecules. On the other hand, X-ray photographs of the PTFE-H specimens show a random orientation of the crystals in all directions, suggesting that the textures are distributed randomly in threedimensional space.

The photographic light scattering patterns for the test specimens are shown in Figures 6 to 8 . The patterns were taken under $H_{\nabla}$ polarizations in which the polarization directions of incident beam and analyzer (placed in between the specimen and the photographic film) are vertical and horizontal, respectively, and under $V_{\mathrm{V}}$ polarizations in which the polarization directions are both vertical. A $\mathrm{He}-\mathrm{Ne}$ gas laser was used as a polarized light source. 


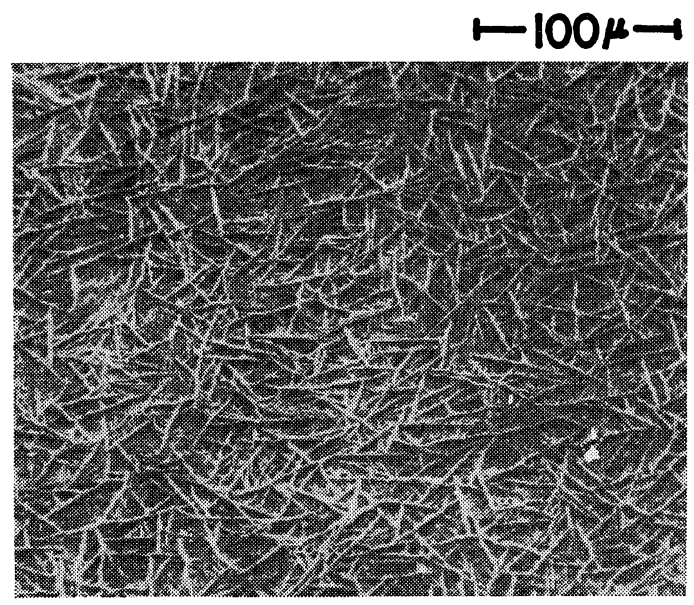

(a)

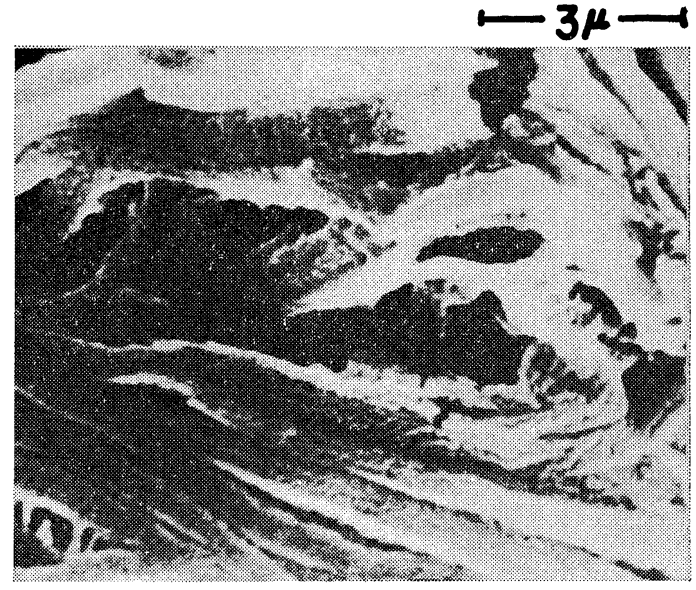

(b)

Figure 3. Scanning electron micrographs of the exterior surface of the PTFE-L specimens; (a) low and (b) high magnifications. Note the striations parallel to the long direction of the band seen in the micrograph (b).

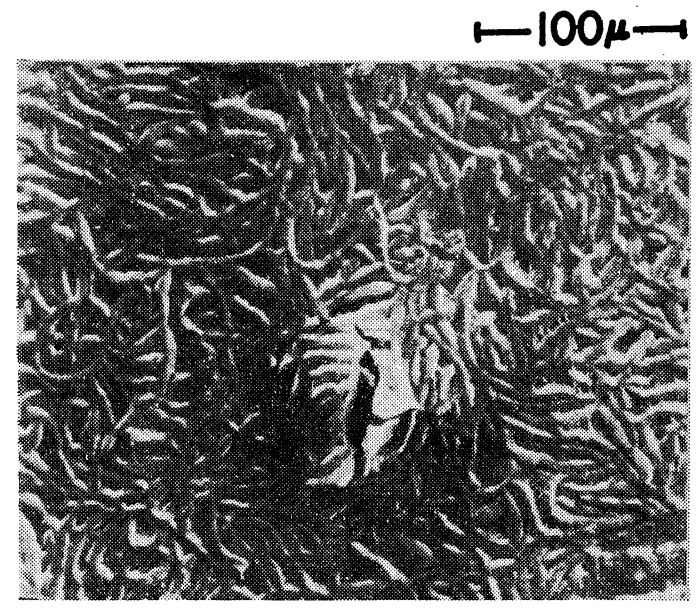

(a)

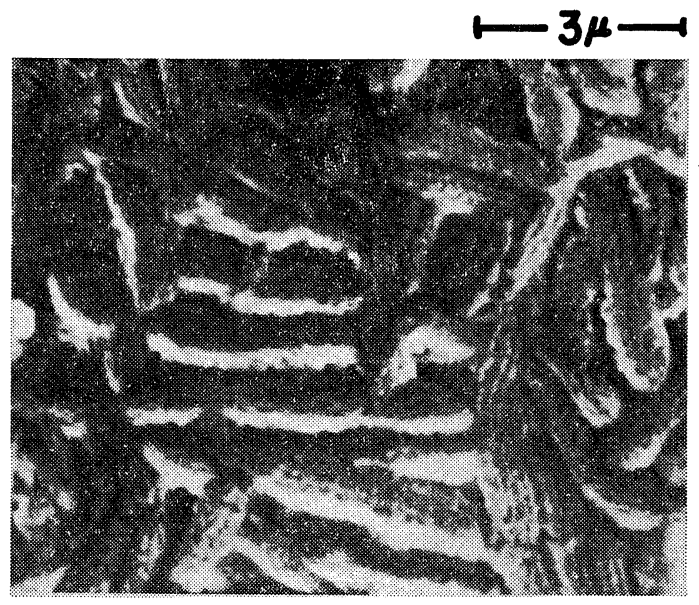

(b)

Figure 4. Scanning electron micrographs of the exterior surface of the PTFE-H specimens; (a) low and (b) high magnifications. Note the striations parallel to the long direction of the band seen in the micrograph (b).

It has been shown that $H_{\mathrm{V}}$ scattering depends upon the orientation fluctuation of the optic axes of anisotropic scattering elements constituting the scattering entity, while $V_{\mathrm{V}}$ scattering depends upon the density fluctuation as well., ${ }^{1,2}$ The intensities of the $H_{\mathrm{V}}$ scattering patterns continuously decrease with increasing scattering angle and vary with fourfold symmetry with respect to the azimuthal angle $\mu$, indicating that these scatterings are typical of the rod-like scattering as seen in Figures 6 to 8. The angular dependence of the $V_{V}$ scattering with respect to $\mu$ depends upon the relative contribution of the orientation and density fluctuations to the scattering. If the density contribution is predominant, the $V_{V}$ pattern becomes independent of $\mu$, while if the orientation contribution is predominant, the $V_{\mathrm{V}}$ pattern becomes dependent on $\mu$. 


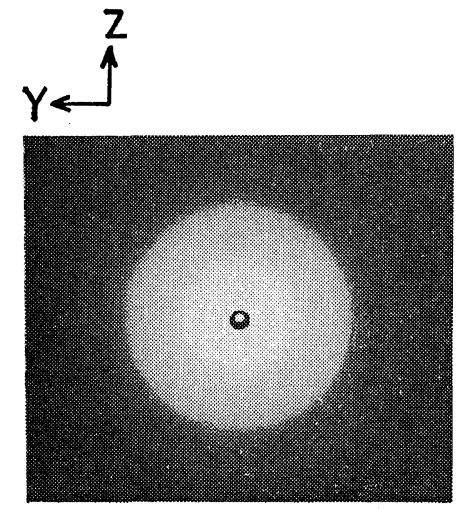

(a)

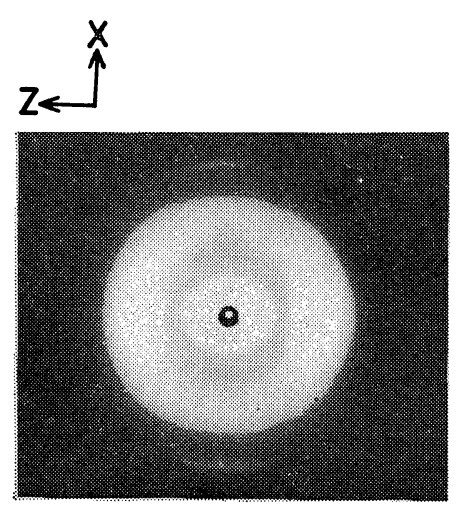

(b) $-\mathrm{i}$
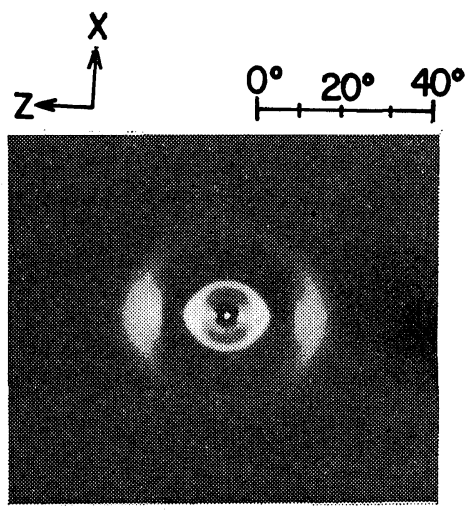

(b)-ii

Figure 5. Wide angle $\mathrm{X}$-ray photographs of the collagen films (specimen C-2). Similar results are also observed for the specimens $\mathrm{C}-1$ and $\mathrm{C}-3$. The patterns are taken with X-ray perpendicular (a). and parallel (b) to the film surfaces oriented in horizontal direction of the figure. The pattern (b)-i was taken with a longer exposure time than the pattern (b)-ii.

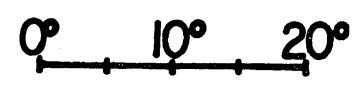

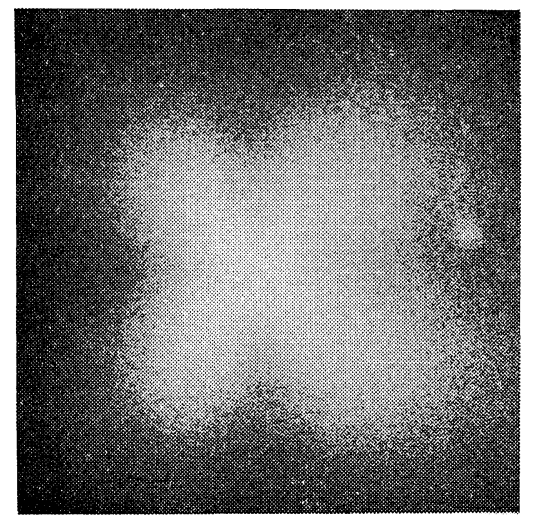

(a)

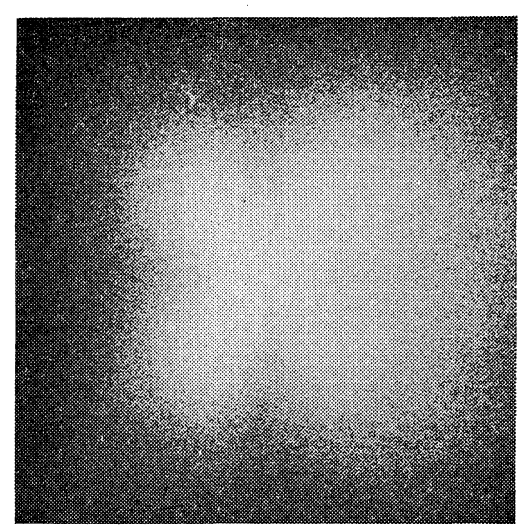

(c)

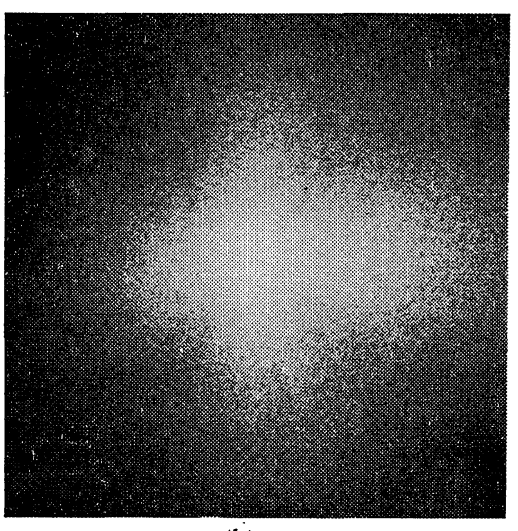

(b)

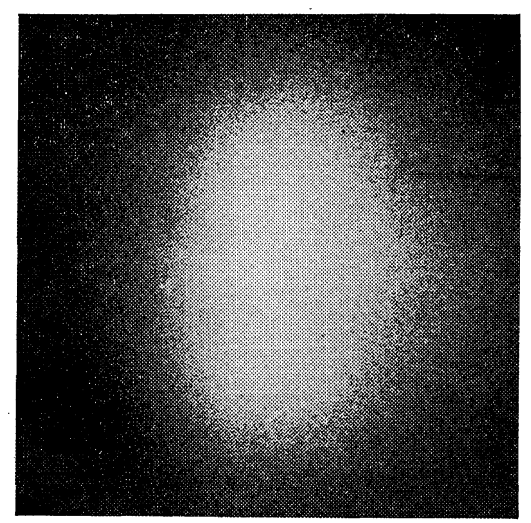

(d)

Figure 6. $H_{\mathrm{V}}$ (a) and $V_{\mathrm{V}}$ (b) scattering patterns of the PTFE-L specimens, and those (c) and (d) of the immersed PTFE-L specimens by $\left(\mathrm{CF}_{2}-\mathrm{CFCl}\right)_{n=2-5}$. The scattering angles are indicated in the top of the figure. 
Light Scattering from Polymer Films Having a Disordered Anisotropic Rod-Like Texture

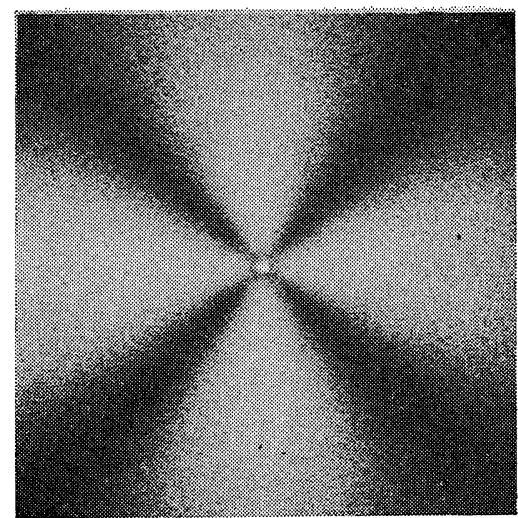

(a)

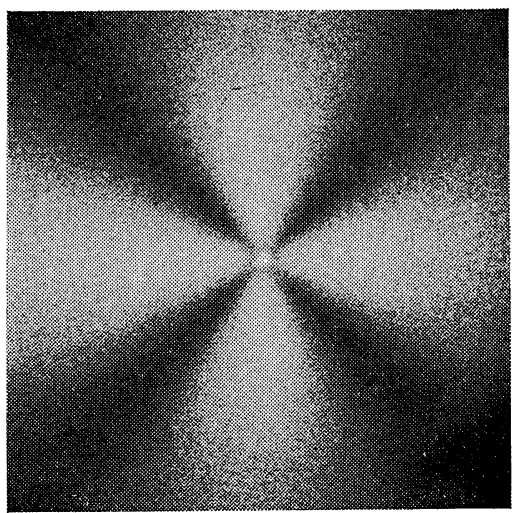

(c)

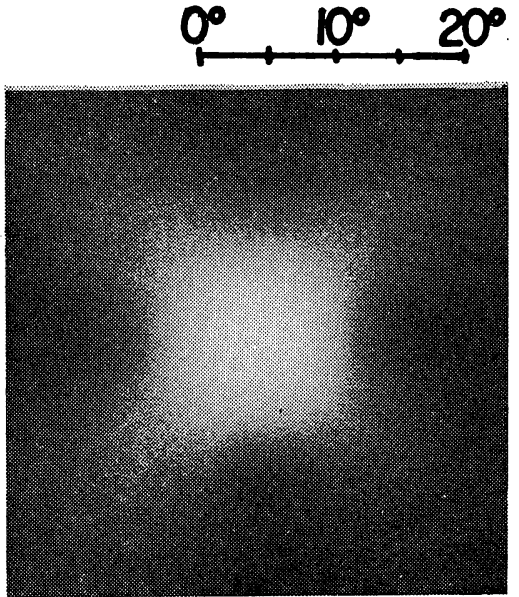

(b)

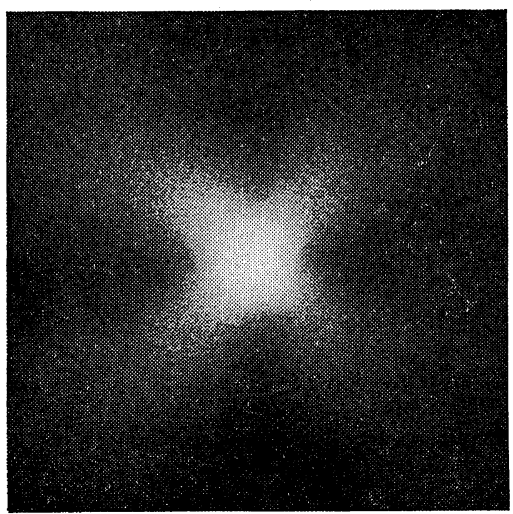

(d)

Figure 7. $H_{\mathrm{V}}$ (a) and $V_{\mathrm{V}}$ (b) scattering patterns of the PTFE-H specimens, and those (c) and (d) of the immersed PTFE-H specimens by $\left(\mathrm{CF}_{2}-\mathrm{CFCl}\right)_{n=2-5}$.

\section{INTERPRETATIONS OF SCATTERING PATTERNS WITH PREVIOUS MODELS}

Important features of the rod-like scattering patterns as shown in Figures 6 to 8 have been qualitatively explained in the previous papers in terms of simple models based upon a random assembly of anisotropic rods (i) with finite length $L$ and infinitesimally thin width in two- ${ }^{1}$ or three-dimensional space, ${ }^{2}$ (ii) with finite length $L$ and finite width of radius $R$ (rods or disks) in three-dimensional space. ${ }^{5}$

In Figure 9 is shown the anisotropic rod model as a scattering entity of the rod-like scattering. The rod is composed of uniaxially anisotropic scattering elements with polarizabilities $\alpha_{\|}$and $\alpha_{\perp}$ along and perpendicular to their principal optical axes whose unit vectors are defined by $\boldsymbol{d}$. The optical axes make a polar angle $\omega_{0}$ with respect to the rod axis $\boldsymbol{r}$ in a special plane defined by an angle $\gamma$ with respect to the cartesian coordinate $O X Y Z$ where the $O X$ axis is parallel to the propagation direction of the incident beam (the unit vector of which is defined as $s_{0}$ ), and the $O Z$ axis is parallel to the vertical direction of the apparatus.

Orientation of the rod is assumed to be random with respect to the angles $\alpha, \beta$, and $\gamma$ for the rods in three-dimensions and with respect to the angle $\alpha$ under $\beta=\gamma=0^{\circ}$ for the rods in twodimensions. The vector $s^{\prime}$ is a unit vector along the scattered light whose intensity is calculated 
T. Hashimoto, Y. Murakami, N. Hayashi, and H. Kawai

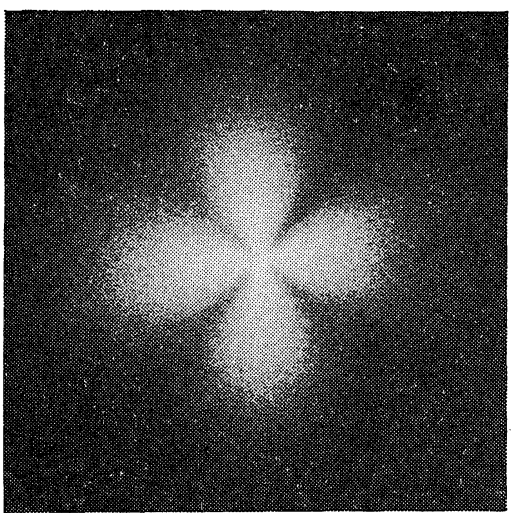

(a)

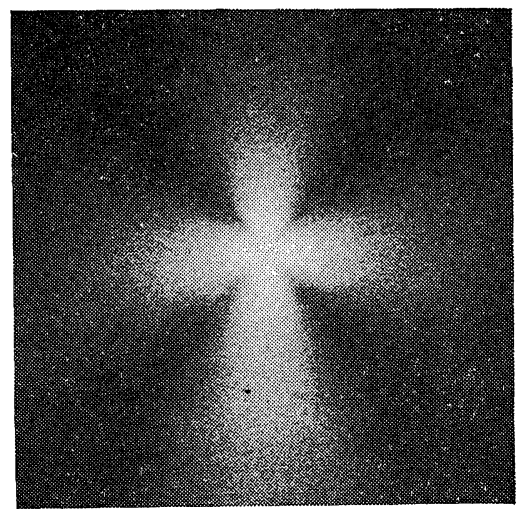

(c)

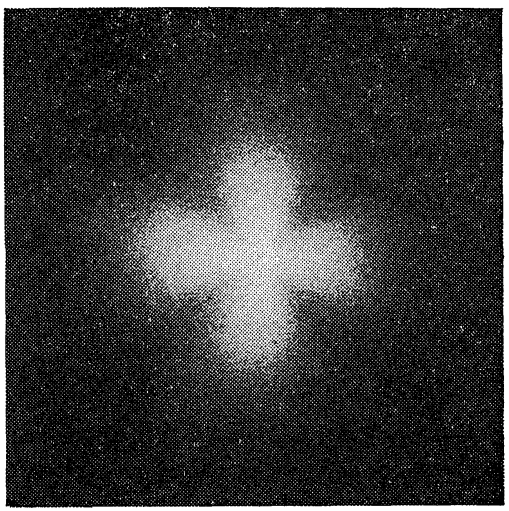

(e)

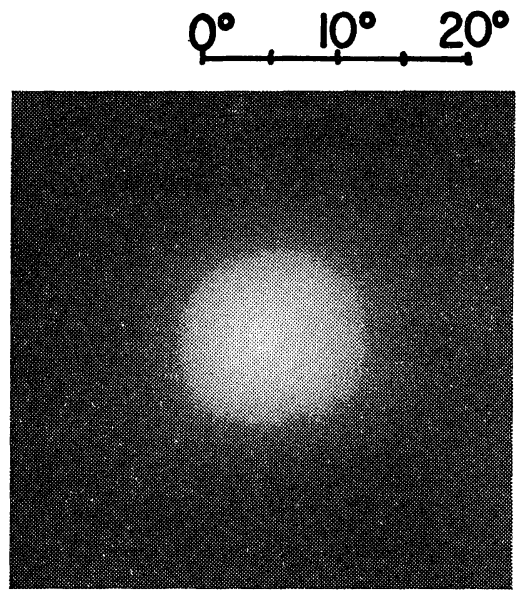

(b)

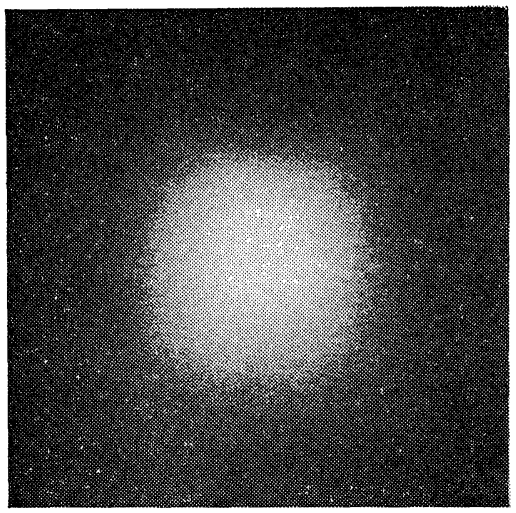

(d)

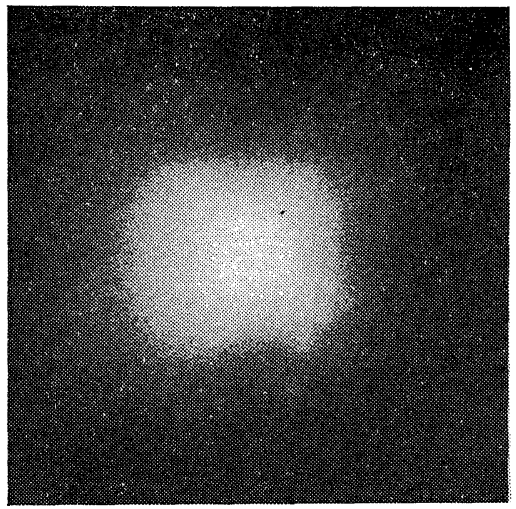

(f)

Figure 8. $H_{\mathrm{V}}$ (left) and $V_{\mathrm{V}}$ (right) scattering patterns of the collagen films; (a), (b) C-1, (c), (d) C-2, and (e), (f) C-3 specimens. 


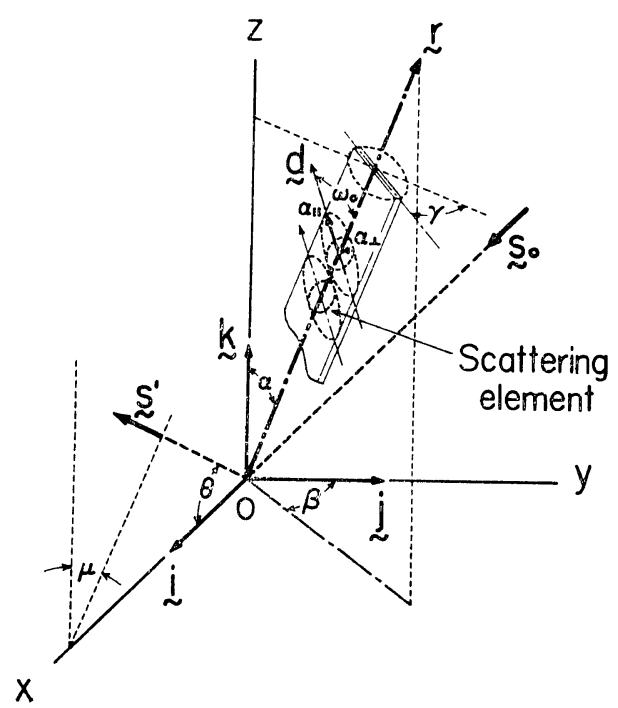

Figure 9. The anisotropic rod model to explain the rod-like scattering.

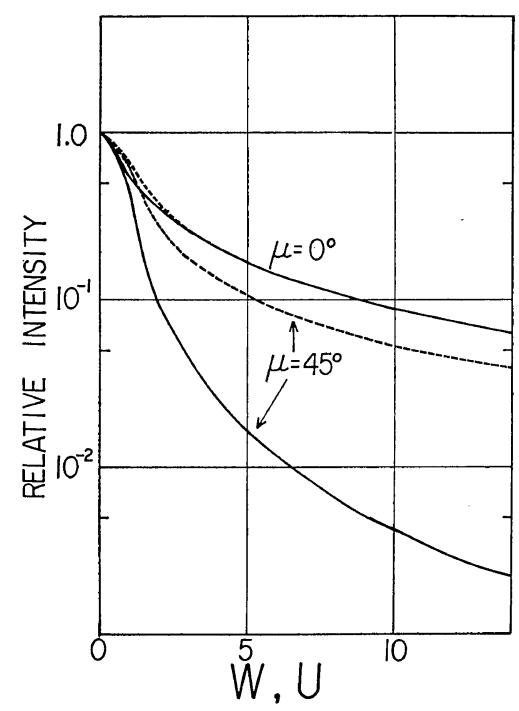

Figure 10. Theoretical $H_{\mathrm{V}}$ scattering intensity distributions of the one-dimensional rods with $\omega_{0}=$ $45^{\circ}$ oriented randomly in two-dimensional space (solid line) and those of the one-dimensional rods with $\omega_{0}=55^{\circ}$ oriented randomly in three-dimensional space (broken line) at $\mu=0$ and $45^{\circ}$. The distributions are plotted as a function of the reduced scattering angle $W=(\pi L / \lambda) \sin \theta$ for the two-dimensional orientation or $U=(2 \pi L / \lambda) \sin (\theta / 2)$ for the three-dimensional orientation. as a function of the scattering angle $\theta$ and azimuthal angle $\mu$.

Important assumptions were involved in these models; (i) the rods were assumed to be identical and homogeneous so that quantities $\omega_{0}, \gamma, \alpha_{/ /}$, and $\alpha_{\perp}$ as well as the dimensions of the rods were kept constant, and (ii) the rods were assumed to be isolated so that an interrod interference effect on the scattering was neglected in the theory. In relation to the assumption (ii), the rods were assumed to be dispersed in an isotropic medium with average polarizability $\alpha_{\mathrm{s}}$.

In spite of the assumptions involved in the calculations, the theoretical results qualitatively accounted for the important features of the experimental scattering patterns. The theoretical intensities continuously decrease with increasing $\theta$ as shown in Figure 10, the rate of which depends upon the size of the rods.

The $\mu$-dependences of the scattering patterns depend upon the angle $\omega_{0}$; the $H_{\mathrm{V}}$ patterns are the $\times$-type for $\omega_{0}$ from $0^{\circ}$ to $\left(\omega_{01}\right)_{\mathrm{c}}$ and for $\omega_{0}$ from $\left(\omega_{02}\right)_{\mathrm{c}}$ to $90^{\circ}$, the + -type patterns for $\omega_{0}$ from $\left(\omega_{01}\right)_{\mathrm{c}}$ to $\left(\omega_{02}\right)_{\mathrm{c}}$ and the circular patterns for $\omega_{0}=\left(\omega_{01}\right)_{\mathrm{c}}$ and $\left(\omega_{02}\right)_{\mathrm{c}}$. The critical values of $\omega_{0},\left(\omega_{01}\right)_{\mathrm{c}}$ and $\left(\omega_{02}\right)_{\mathrm{c}}$, at which the patterns change their appearance depend upon the models; $\left(\omega_{01}\right)_{\mathrm{c}}=$ $30^{\circ} 33^{\prime}$ and $\left(\omega_{02}\right)_{\mathrm{c}}=70^{\circ} 07^{\prime}$ for the assembly of the one-dimensional rods in three-dimensional space, and $\left(\omega_{01}\right)_{\mathrm{c}}=22^{\circ} 30^{\prime}$ and $\left(\omega_{02}\right)_{\mathrm{c}}=67^{\circ} 30^{\prime}$ for the assembly of the one-dimensional rods intwo-dimensional space. ${ }^{2}$ The $\times$-type patterns have maximum and minimum intensities at odd and even multiples of $\mu=45^{\circ}$, respectively, and the + -type patterns have maximum and minimum intensities at even and odd multiples of $\mu=45^{\circ}$, respectively, while the circular type patterns are independent of $\mu$.

Although the quantitative angular dependences of the patterns with respect to $\theta$ and $\mu$ are different for different models as seen in Figure 10, the qualitative appearances of the patterns are suggested to be almost the same for these models from the previous works. ${ }^{1-3}$

The $V_{V}$ scattering patterns depend upon the anisotropy term $\left(\alpha_{\|}-\alpha_{\perp}\right)$ as well as the density term $\left(\alpha-\alpha_{\mathrm{s}}\right)$ where $\alpha$ is an average polarizability of the rods defined by $\alpha=\left(\alpha_{\|}+2 \alpha_{\perp}\right) / 3$, so that the term $\left(\alpha-\alpha_{\mathrm{s}}\right)$ is related to the difference of the densities (polarizabilities) between the rods 
and the surrounding medium. If the value of $\left(\alpha-\alpha_{\mathrm{s}}\right)$ is much larger than $\left(\alpha_{\|}-\alpha_{\perp}\right)$, the $V_{\mathrm{V}}$ patterns become circular patterns which are independent of $\mu$. On the other hand, if the value of $\left(\alpha-\alpha_{\mathrm{s}}\right)$ is much smaller than $\left(\alpha_{\|}-\alpha_{\perp}\right)$, then the patterns are angularly dependent with respect to $\mu$, the dependences of which are predicted by the value of $\omega_{0} .{ }^{1-3}$

In view of the theoretical results, the experimental $\times$-type $H_{\mathrm{V}}$ patterns shown in Figure 6 for the PTFE-L specimens are interpreted as those from the rod-like texture with $\omega_{0}$ nearly equal to 0 or $90^{\circ}$. Unique determination of the possible value of $\omega_{0}$ is not possible by the light-scattering method alone, but may be made with the aid of microscopic observations (Figure 1a), from which the value of $\omega_{0}$ appears to be nearly $90^{\circ}$. The $V_{\mathrm{V}}$ pattern for the dry specimens is two-fold symmetric with maximum intensities at $\mu=90$ and $180^{\circ}$ as shown in Figure 6b. The $\mu$-dependence, however, is weak and not clear, indicating that the density contribution is not negligibly small compared with the orientation contribution.

Upon immersing the specimen in the liquid, the $H_{\mathrm{V}}$ pattern does not change, indicating that the texture itself is not modified, while the $V_{\mathrm{V}}$ pattern changes in terms of the $\mu$-dependence. The $\mu$-dependence becomes stronger and clearer upon immersion, indicating that the anisotropy contribution becomes more important than the density contribution upon immersing, and that the difference of refractive indices (or polarizabilities) between the texture and the surrounding becomes smaller on the penetration of the liquid into the specimen.

On the other hand, the +-type $H_{\mathrm{V}}$ scattering patterns as observed for the PTFE-H and denatured collagen films should be interpreted as those from the textures with $\omega_{0}$ in the range of $\left(\omega_{01}\right)_{\mathrm{c}}$ and $\left(\omega_{02}\right)_{\mathrm{c}}$ (Figures 7 and 8 ). The $\times$-type $V_{\mathrm{V}}$ patterns observed for the PTFE-H specimens further restrict the possible values of $\omega_{0}$, which are, according to the previous work, ${ }^{2}$ nearly $55^{\circ}$. This fact appears to indicate that the chain axes are inclined to the base of the crystal lamella ${ }^{3}$ rather than normal to it as in the case of PTFE$\mathrm{L}$ specimens and as observed by Bunn, et al. ${ }^{17}$

It is suggested that, in case of the collagen films, the tropocollagen molecules were packed side-by-side with the angle $\omega_{0}$ within the texture. ${ }^{3}$ It should be noted that the arguments are valid when the optical axes are parallel to the molecular chain axes of the PTFE specimens and to the tropocollagen molecules. Detailed morphological observations, however, have not been made up to now for the denatured collagen films. The observations would be important for future study.

The $\mu$-dependence of $V_{\mathrm{V}}$ scattering pattern of the PTFE-H specimens becomes sharp when the specimens are immersed in liquid, suggesting again that, upon immersion, the anisotropy contribution becomes relatively greater (Figures $7 b$ and d). The $V_{\mathrm{V}}$ patterns from the denatured collagen films are the $\times$-patterns with very little $\mu$-dependence, indicating that the density contribution to the scattering is large (Figure 8). The change of the $V_{V}$ patterns upon swelling the specimens in saline solution was discussed in the previous paper. $^{2}$

\section{A QUANTITATIVE TEST OF THE PREVIOUS MODELS WITH INTENSITY DISTRIBUTIONS}

For the quantitative test, the experimental intensity distributions were compared with the theoretical intensity distributions (Figure 10) based upon the random assembly of the onedimensional rods. The intensity distributions were photometrically measured by using an apparatus constructed in this laboratory. The details of the apparatus will not be discussed here but are similar to those constructed by Hashimoto and Stein, et al. ${ }^{24}$ except for the fact that $\mathrm{He}-\mathrm{Ne}$ gas laser was used in this apparatus rather than a mercury lamp as a light source.

In Figure 11 are shown the results of the photometric measurements of the collagen films at $\mu=0$ and $45^{\circ}$ as a function of $\theta$ under $H_{\nabla}$ polarization conditions, and in Figure 12 are shown contour plots of experimental $H_{\mathrm{V}}$ scatterings for the collagen films. In Figure 13 are shown the experimental $H_{\mathrm{V}}$ intensity distributions at $\mu=0$ and $45^{\circ}$ for the PTFE specimens. The experimental intensity distributions agree with the theoretical intensity distributions in qualitative appearances that (i) the intensities continuously decrease with increasing scattering 


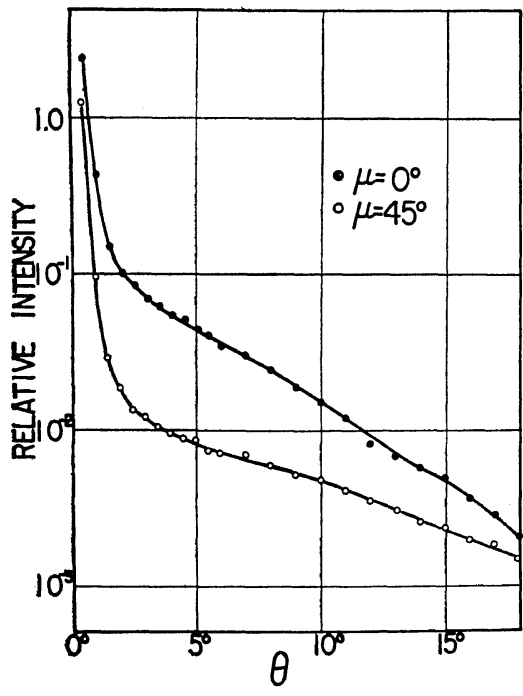

(a)

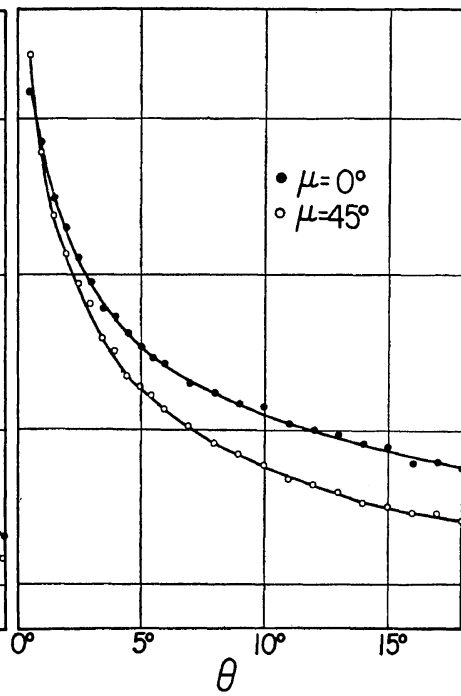

(b)

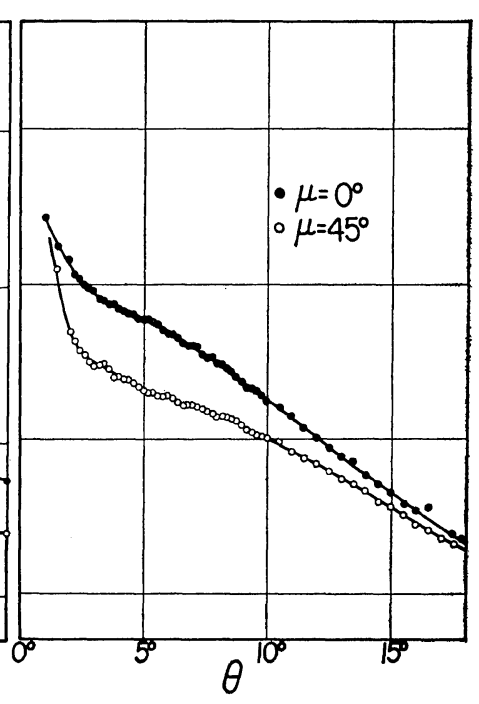

(c)

Figure 11. The experimental $H_{\mathrm{V}}$ scattering intensity distributions of the collagen films at $\mu=0$ and $45^{\circ}$, (a) C-1, (b) C-2 and (c) C-3 specimens.

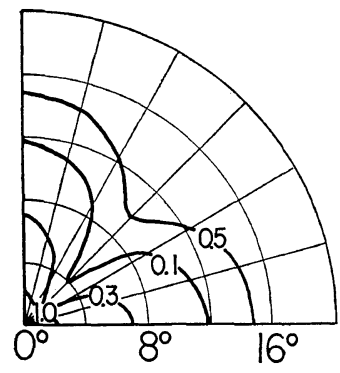

(a)

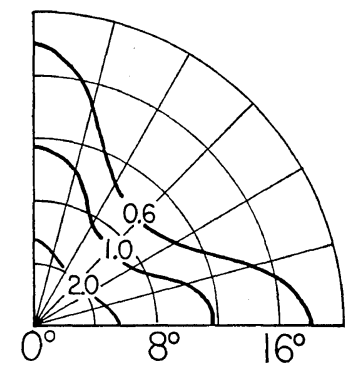

(b)

Figure 12. Contour plots of the experimental $H_{\mathrm{V}}$ scattering intensity distributions of the collagen films; (a) C-1 and (b) C-2 specimens. The contour plot of the specimen C-3 is similar to that of C-1.

angles and that (ii) intensities have maxima at odd multiples of $\mu=45^{\circ}$ for the PTFE-L specimens, and at integral multiples of $\mu=90^{\circ}$ for other specimens.

One can see, however, by comparing quantitatively the theoretical and experimental results, that the theoretical results for the assembly of one-dimensional rods deviate from the experimental results in the following respects. (i) The $\mu$-dependences of the theoretical intensities continuously increase with increasing scattering angles, while those of the experimental intensities do not do so for the PTFE-H (Figure 13b) and

the collagen C-1 and C-3 specimens (Figures 11a and c, and Figure 12b). The discrepancy is especially remarkable at large scattering angles. (ii) The theory deviates also at very small scattering angles where the experimental scattered intensity decreases with increasing scattering angles at a greater rate than the theoretical intensity (Figures 10 and 11).

The discrepancies may be interpreted in terms of the principle of the reciprocal phenomenon ${ }^{26}$ which interrelates the angular distribution of scattering to the size of scattering matter or to the size of a correlated region of the anisotropy and orientation fluctuations (in case of $H_{\mathrm{V}}$ scattering) of the matter.

The rapid decrease of the experimental intensity at small angles may be interpreted as arising from the fluctuations correlated over a larger distance than the fluctuations expected theoretically for the isolated rods. In fact, so far as we have studied, it was not possible to explain the discrepancy either by increasing the rod length $L$ or by introducing an effect of finite lateral dimensions into the isolated models. ${ }^{5,6}$ Therefore the discrepancy may arise from an effect of interparticle interference of the scattered waves which is ignored in the isolated rod theory. The interference involves the scattering from a 


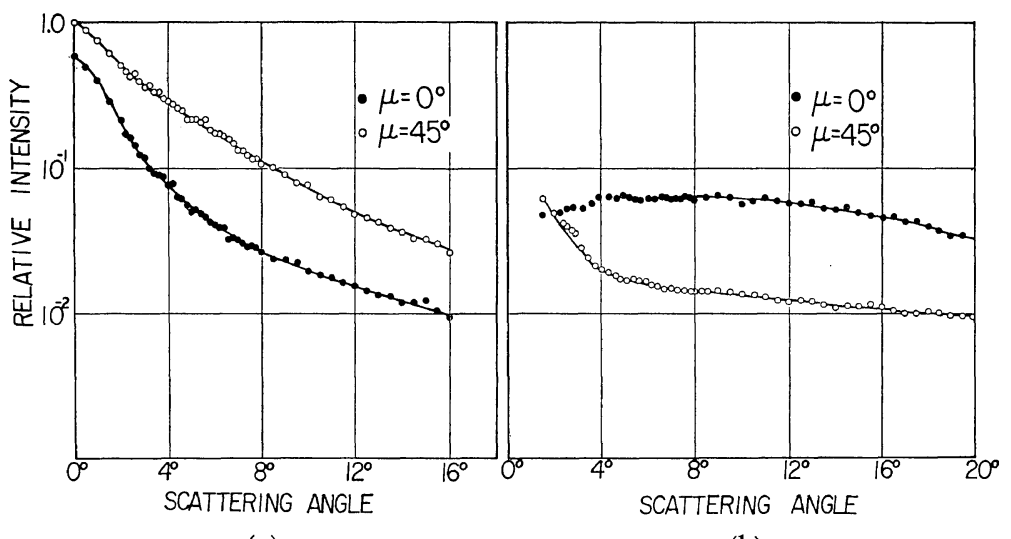

(a)

(b)

Figure 13. The experimental $H_{\mathrm{V}}$ scattered intensity distributions at $\mu=0$ and $45^{\circ}$ for the PTFE-L (a) and PTFE-H (b) specimens.

region composed of a coherent assembly of the textures, i.e., from the correlated region which is bigger than that of the isolated rod-like texture.

On the other hand, the discrepancy at high scattering angles may be attributed to a deviation of the model from the real texture in terms of fluctuations correlated over a shorter distance than the rods themselves. Inhomogeneities of the rod in terms of anisotropy and orientation of optical axes of the anisotropic scattering elements may be a possible source which accounts for the discrepancy, since they involve fluctuations correlated over a shorter distance than those expected for the isolated rods. In next section we shall discuss the effect of the internal fluctuations of the anisotropy and orientation on the rod-like scattering which was neglected in the previous papers.

Another possibility which may account for the discrepancy at high scattering angles may be that the lateral dimensions of the rod are not, in general, infinitesimaly small as assumed in the theory but are finite. The effect of the finite lateral dimensions on the scattering was discussed previously for cylindrical particles ${ }^{5}$ and will be discussed in a subsequent paper for anisotropic rectangular plates. $^{6}$ The effect produced the experimental tendency that the $\mu$-dependences of the intensity decrease with increasing $\theta$ in a manner somewhat different to the effect of the internal disorders as will be discussed elsewhere. ${ }^{6}$

\section{EFFECT OF POLYDISPERSITY IN SIZE OF THE ROD-LIKE TEXTURE}

The effect of the polydispersity was considered in order to understand the origin of the discrepancy at high scattering angles.

The amplitude of scattering $(E(\alpha))$ from the anisotropic rod with infinitesimally thin lateral dimensions oriented by an angle $\alpha$ from the vertical direction $(O Z)$ is given, according to Rayleigh-Gans theory, by

$$
E(\alpha)=C \int_{-L / 2}^{L / 2}(\boldsymbol{M} \cdot \boldsymbol{O}) \exp [i k(\boldsymbol{r} \cdot s)] \mathrm{d} \boldsymbol{r}
$$

where $L$ is the length of the $\operatorname{rod}$, and $r$ is a vector along the rod axis (Figure 14). The vector $s$ is a vector defined by $\left(s_{0}-s^{\prime}\right)$ where $s_{0}$ and $s^{\prime}$ are unit vectors parallel to the propagation directions of the incident and scattered beams, respectively. The quantity $C$ is a constant related to an absolute intensity of the scattering, and $k$ is defined by $2 \pi / \lambda$, where $\lambda$ is the wavelength of light in the medium. The vectors $\boldsymbol{M}$ and $\boldsymbol{O}$ are, respectively, the induced dipolemoment of the scattering element located at $\boldsymbol{r}$ in the rod, and a unit vector along the polarization direction of the analyzer set perpendicular to $s^{\prime}$ and inbetween the sample and detector to record the scattering.

The induced dipolemoment $\boldsymbol{M}$ for vertically polarized incident beam is given by

$$
\begin{aligned}
\boldsymbol{M} & =E_{0}\left[\delta_{0}(\boldsymbol{k} \cdot \boldsymbol{d}) \boldsymbol{d}+\boldsymbol{b}_{\mathrm{t}} \boldsymbol{k}\right] \\
\boldsymbol{d} & =\boldsymbol{j} \sin \left(\alpha+\omega_{0}\right)+\boldsymbol{k} \cos \left(\alpha+\omega_{0}\right)
\end{aligned}
$$




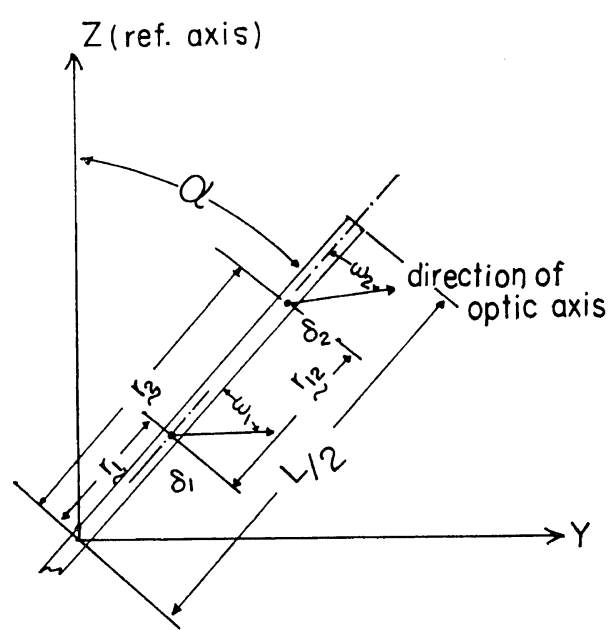

Figure 14. The model of one-dimensional disordered rods oriented randomly in the two-dimensional space of $O Y Z$.

where $E_{0}$ is the field strength of incident beam, and $\delta_{0}$ is the anisotropy of the scattering element defined by $\left(\alpha_{\|}-\alpha_{\perp}\right)$ which is assumed to be uniaxially symmetrical with polarizabilities $\alpha_{\|}$ and $\alpha_{\perp}$ along and perpendicular to the optical axis. The vector $\boldsymbol{d}$ is a unit vector along the optical axis and is assumed to lie in the plane of $O Y Z$ and with an angle $\omega_{0}$ with respect to $r$. The unit vector $\boldsymbol{O}$ for the $H_{\nabla}$ polarization is approximately given, at small $\theta,{ }^{20}$ by $\boldsymbol{O}=\boldsymbol{j}$. Hence for the $H_{\nabla}$ polarization, $(M \cdot O)$ is given by

$$
(\boldsymbol{M} \cdot \boldsymbol{O})_{H_{V}}=(1 / 2) \delta_{0} \sin 2\left(\alpha+\omega_{0}\right)
$$

If the rod is homogeneous, then $(\boldsymbol{M} \cdot \boldsymbol{O})_{H_{V}}$ is independent of $r$, so that $(\boldsymbol{M} \cdot \boldsymbol{O})_{H_{V}}$ is put outside the integral in eq 1. The integral over $r$ is easily carried out to result in

$$
\begin{aligned}
{[E(\alpha)]_{H_{\mathrm{V}}} } & =\frac{1}{2} C L \delta_{0} \sin 2\left(\alpha+\omega_{0}\right) \frac{\sin [W \cos (\alpha-\mu)]}{W \cos (\alpha-\mu)} \\
W & =(\pi L / \lambda) \sin \theta
\end{aligned}
$$

Consequently, the $H_{\mathrm{V}}$ scattered intensity $\left(I_{H_{\mathrm{V}}}\right)$ for the random assembly of rods is given, assuming that the interparticle interference is negligible, by

$$
I_{H_{\mathrm{V}}}=K \int_{0}^{2 \pi}[E(\alpha)]_{H_{\mathrm{V}}}[E(\alpha)]_{H_{\mathrm{V}}}^{*} \mathrm{~d} \alpha=K \int_{0}^{2 \pi}[E(\alpha)]_{H_{\mathrm{V}}}^{2} \mathrm{~d} \alpha
$$

The results of numerical calculations of eq 5 are shown in Figure 10 for $\omega_{0}=45^{\circ}$ (solid line).

The eq 5 assumes that the size of the rod is uniform. It is likely that there is polydispersity in rod size in real systems. In this care an average $H_{\mathrm{V}}$ scattered intensity is given by

$$
I_{H_{\mathrm{V}}}=\int_{0}^{\infty} N(L) I_{H_{\mathrm{V}}}(L) \mathrm{d} L / \int_{0}^{\infty} N(L) \mathrm{d} L
$$

where $N(L)$ is number of the rods having length $L$. In this paper, the effect was considered by assuming a box distribution with upper and lower limits of the length being $L-\varepsilon$ and $L+\varepsilon$ for the function $N(L)$ where $\varepsilon$ is a parameter describing the polydispersity.

For the homogeneous rod, the average $H_{\nabla}$ intensity is given, from eq 5 , by

$$
\begin{aligned}
I_{H_{\mathrm{V}}}(L)= & K_{1}\left(L / W_{1}\right)^{2}\left\{W_{1}\left[I_{1}\left(W_{1}\right)-J_{1}\left(W_{1}\right)\right]\right. \\
& +2 \cos 4 \xi_{0}\left[-12 J_{2}\left(W_{1}\right)+W_{1} J_{1}(W)\right. \\
& \left.\left.-4 J_{0}\left(W_{1}\right)+4-W_{1} I_{2}\left(W_{1}\right)\right]\right\}
\end{aligned}
$$

where $W_{1}=2 W, \xi_{0}=\mu+\omega_{0}, K_{1}=C^{2} \delta_{0}^{2} / 2$ and

$$
I_{1}\left(W_{1}\right)=\int_{0}^{W_{1}} J_{0}(x) \mathrm{d} x, \quad I_{2}\left(W_{1}\right)=\int_{0}^{W_{1}} J_{4}(x) \mathrm{d} x
$$

Hence it follows that

$$
\begin{aligned}
& I_{H_{\mathrm{V}}}\left(\mu=0^{\circ}\right)-I_{H_{\mathrm{V}}}\left(\mu=45^{\circ}\right) \\
&= K_{1} \cos 4 \omega_{0}\left[-12 J_{2}\left(W_{1}\right)+W_{1} J_{1}\left(W_{1}\right)\right. \\
&\left.-4 J_{0}\left(W_{1}\right)+4-W_{1} I_{2}\left(W_{1}\right)\right]
\end{aligned}
$$

and

$$
\begin{aligned}
{\left[I_{H_{\mathrm{V}}}(\mu\right.} & \left.\left.=0^{\circ}\right)+I_{H_{\mathrm{V}}}\left(\mu=45^{\circ}\right)\right] / 2 \\
& =K_{1} W_{1}\left(L / W_{1}\right)^{2}\left[I_{1}\left(W_{1}\right)-J_{1}\left(W_{1}\right)\right]
\end{aligned}
$$

From eq 9 it is seen that the arithmetric average of the $H_{\mathrm{V}}$ intensities at $\mu=0^{\circ}$ and $45^{\circ}$ is independent of the value of $\omega_{0}$ but is dependent only upon the length $L$ or the average length $L$. Therefore upon analyzing the average, the length $L$ may be obtained independently from the value of $\omega_{0}$. The value of $\omega_{0}$ may be also obtained from eq 8 and 9 . It should be noted that the difference between the intensities at $\mu=0$ and $45^{\circ}$, i.e., $\left|I_{H_{\mathrm{V}}}\left(\mu=0^{\circ}\right)-I_{H_{\mathrm{V}}}\left(\mu=45^{\circ}\right)\right|$ is a maximum for $\omega_{0}=(n \pi / 4)$, i.e., $\omega_{0}=0^{\circ}, 45^{\circ}$, and etc., and zero for $\omega_{0}=(2 m+1) \pi / 2$, i.e., $\omega_{0}=$ $22.5,67.5^{\circ}$, and etc. It should be also noted that the term of $\cos 4 \xi_{0}$ in eq 7 should be replaced by the term of $\left(\cos 4 \omega_{0} \cos 4 \mu\right)$ if positive and negative values of $\omega_{0}$ are equally probable. 


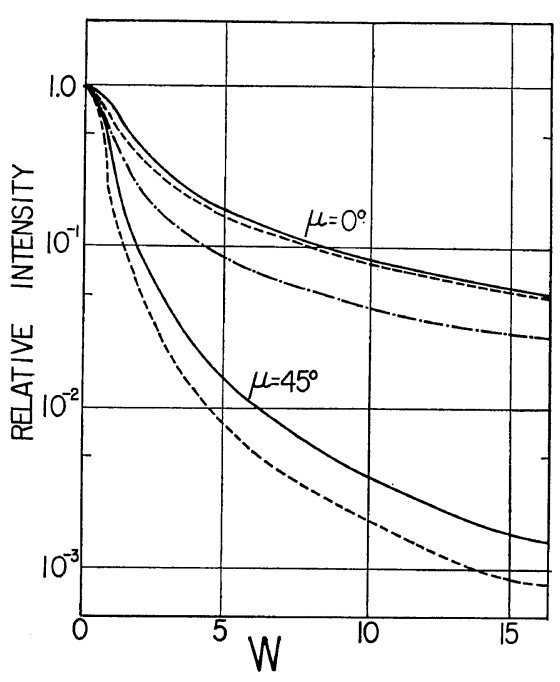

Figure 15. The effect of polydispersity of the rod size for the two-dimensional distributions of the one-dimensional rods. The solid and broken curves are those for the monodispersed and box distributions. The chain line curve corresponds to the arithmetric average.

The eq 8 and 9 are still valid even in this case.

On the other hand, for the random assembly of the one-dimensional rod oriented in threedimensional space, the value of $\mid I_{H_{\mathrm{V}}}\left(\mu=0^{\circ}\right)-$ $\left.I_{H_{\mathrm{V}}}\left(\mu=45^{\circ}\right)\right\}$ is a maximum for $\omega_{0} \cong 50^{\circ}$, and the arithmetric average still depends upon $\omega_{0}$ as expected from the previous results. ${ }^{2}$ The $H_{\mathrm{V}}$ scattering from the system was also plotted in Figure 10 (broken line) for the case of $\omega_{0}=55^{\circ}$, the value of which is close to the one giving rise to maximum $\mu$-dependence. It is seen that the $\mu$-dependence is smaller for the three-dimensional distribution than for the two-dimensional distribution.

In Figure 15 is shown the effect of polydispersity of the rod size for $\omega_{0}=45^{\circ}, L=40 / \lambda$, and $\varepsilon=40 / \lambda$ for the two-dimensional distribution of the rod. The effect is very small and appreciable only at $\mu=45^{\circ}$. The arithmetric average of the intensities becomes almost identical to that of the identical rod, so that the polydispersity of the rod size would not account for the discrepancy between theoretical and experimental intensities discussed earlier.

\section{EFFECT OF INTERNAL DISORDERS OF THE ROD-LIKE TEXTURE}

In order to study the effect of internal disorders, calculations of $H_{\mathrm{V}}$ scattering were performed for the one-dimensional rods oriented randomly in two-dimensional plane perpendicular to propagation direction of the incident beam. The effect of the polydispersity of the rod size was ignored from the previous conclusion. The effect of the disorders for the one-dimensional rods oriented randomly in three-dimensional space is suggested to be qualitatively similar to that for the rods oriented randomly in two-dimensional space from the previous work. ${ }^{1,2}$ The calculations were performed in a manner similar to those of the disordered spherulites. ${ }^{21-23}$.

If the rod is inhomogeneous, then the anisotropy of the scattering element $\delta$ and the orientation of the optical axis of the scattering element $\omega$ fluctuate with $r$ from their average values $\delta_{0}$ and $\omega_{0}$, respectively. Hence $(\boldsymbol{M} \cdot \boldsymbol{O})_{H_{V}}$ cannot be put outside the integral in eq 1 . Now from eq 1 and 3 , it follows that

$$
\begin{aligned}
E_{H_{\mathrm{V}}}(\alpha)= & (1 / 2) C \int_{-\infty}^{\infty} \sigma(r)[\delta \sin 2(\alpha+\omega)] \\
& \times \exp [i k(\boldsymbol{r} \cdot s)] \mathrm{d} r
\end{aligned}
$$

where $\sigma(r)$ is a shape factor of the rod defined by

$$
\sigma(r)=\left\{\begin{array}{lll}
1 & \text { for } & |r| \leq L / 2 \\
0 & \text { for } & |r|>L / 2
\end{array}\right.
$$

The quantities $\delta$ and $\omega$ which are functions of $r$ can be written as follows,

$$
\delta(r)=\delta_{0}+\Delta \delta(r), \quad \omega(r)=\omega_{0}+\Delta(r)
$$

where $\Delta \delta(r)$ and $\Delta(r)$ are the local fluctuations of $\delta$ and $\omega$.

From eq 10 , the $H_{\mathrm{V}}$ scattered intensity from a rod oriented at an angle $\alpha$ with respect to $O Z$-axis (Figure 14) is given by

$$
\begin{aligned}
I_{H_{\mathrm{V}}}(\alpha)= & K E_{H_{\mathrm{V}}}(\alpha) E_{H_{\mathrm{V}}}^{*}(\alpha) \\
= & K_{1} \int_{-\infty}^{\infty} \int_{-\infty}^{\infty} \sigma\left(r_{1}\right) \sigma\left(r_{1}+r_{12}\right) \delta_{1} \delta_{2} \sin 2\left(\alpha+\omega_{1}\right) \\
& \times \sin 2\left(\alpha+\omega_{2}\right) \exp \left[i k\left(r_{12} \cdot s\right)\right] \mathrm{d} r_{1} \mathrm{~d} r_{12} \quad(13)
\end{aligned}
$$

where $\delta_{i}$ and $\omega_{i}$ are the anisotropy and the orientation angle of the optic axis of the scattering element located at $r_{i}$ from the center of 
the rod (Figure 14), respectively, and $r_{12}=r_{2}-r_{1}$ and $\boldsymbol{r}_{12}=\boldsymbol{r}_{2}-\boldsymbol{r}_{1}$.

Define $P\left(r_{12}\right)$, the integration over $r_{1}$ keeping $r_{12}$ constant as

$$
\begin{aligned}
P\left(r_{12}\right)= & \int_{-\infty}^{\infty} \sigma\left(r_{12}\right) \sigma\left(r_{1}+r_{12}\right) \delta_{1} \delta_{2} \sin 2\left(\alpha+\omega_{1}\right) \\
& \times \sin 2\left(\alpha+\omega_{2}\right) \mathrm{d} r_{1}
\end{aligned}
$$

Noticing that $\sigma\left(r_{1}\right) \sigma\left(r_{1}+r_{12}\right)$ is not zero only when both $r_{1}$ and $r_{2}$ are within the rod, the function $P\left(r_{12}\right)$ is rewritten as

$$
\begin{aligned}
P\left(r_{12}\right) & =\int_{l\left(r_{12}\right)} \delta_{1} \delta_{2} \sin 2\left(\alpha+\omega_{1}\right) \sin 2\left(\alpha+\omega_{2}\right) \mathrm{d} r_{1} \\
& =l\left(r_{12}\right)\left\langle\delta_{1} \delta_{2} \sin 2\left(\alpha+\omega_{1}\right) \sin 2\left(\alpha+\omega_{2}\right)\right\rangle_{r_{12}}
\end{aligned}
$$

where the integration is carried out over a region of $r_{1}$ satisfying the restriction that both $r_{1}$ and $r_{2}$ are within the rod, and $l\left(r_{12}\right)$ is the length of the region and is obviously given by

$$
l\left(r_{12}\right)=L \Gamma\left(r_{12}\right)=\left\{\begin{array}{lll}
L-\left|r_{12}\right| & \text { for } & \left|r_{12}\right| \leq L \\
0 & \text { for } & \left|r_{12}\right|>L
\end{array}\right.
$$

where $\Gamma\left(r_{12}\right)$ is the one-dimensional correlation function of homogeneous rod of length $L$. The function $\langle F\rangle_{r_{12}}$ denotes an average of the quantity $F$ under a given constant $r_{12}$, and is associated with correlation functions of the internal disorders with $\delta$ and $\omega$. In eq 14, the internal disorders of $\delta$ and $\omega$ are assumed to be random. If they are not random, the function $\langle F\rangle_{r_{12}}$ should depend also upon $r_{1}$ and be replaced by $\langle F\rangle_{r_{1}, r_{12}}$.

The average $\langle F\rangle_{r_{12}}$ can be calcutated in a manner shown in previous papers for the disordered spherulites, ${ }^{21-23}$ the results of which is given by

$$
\begin{aligned}
\left\langle\delta_{1} \delta_{2}\right. & \left.\sin 2\left(\alpha+\omega_{1}\right) \sin 2\left(\alpha+\omega_{2}\right)\right\rangle_{r_{12}} \\
= & (1 / 4) \delta_{0}^{2}\left[1+A \Psi\left(r_{12}\right)\right]\left[\sin ^{2} 2 \xi_{0}\left\langle\cos ^{2} 2 \Delta_{1}\right\rangle_{\mathrm{aV}}\right. \\
& \left.\quad+\cos ^{2} 2 \xi_{0}\left\langle\sin ^{2} 2 \Delta_{1}\right\rangle_{\mathrm{av}}\right] f\left(r_{12}\right)
\end{aligned}
$$

where $\xi_{0}=\alpha+\omega_{0}$, and $\Psi\left(r_{12}\right)$ and $f\left(r_{12}\right)$ are the correlation functions for the internal anisotropy and orientation fluctuations, respectively, and are defined by

$$
\begin{aligned}
\Psi\left(r_{12}\right) & =\left\langle\Delta \delta_{1} \Delta \delta_{2}\right\rangle_{r_{12}} /\left\langle\left(\Delta \delta_{1}\right)^{2}\right\rangle_{\mathrm{av}}, \\
f\left(r_{12}\right) & =\left\langle\cos 2 \Delta_{12}\right\rangle_{r_{12}}
\end{aligned}
$$

The quantity $\Delta_{12}$ is defined by $\Delta_{12}=\Delta_{2}-\Delta_{1}=$ $\omega_{2}-\omega_{1}$, the angle characterizing a relative orientation of the optic axes of two scattering elements separated by a distance $r_{12}$. The quantities $\left\langle\left(\Delta \delta_{1}\right)^{2}\right\rangle_{\mathrm{av}}$ and $\left\langle\sin ^{2} 2 \Delta_{1}\right\rangle_{\mathrm{av}}$ (or $\left\langle\cos ^{2} 2 \Delta_{1}\right\rangle_{\mathrm{av}}$ ) are parameters associated with the magnitudes of the anisotropy and orientation disorders. It is assumed in eq 16 , as in the previous paper, ${ }^{22}$ that the anisotropy and orientation disorders are independent to each other.

Now from eq 13 to 16 , it follows that

$$
\begin{aligned}
I_{H \mathrm{~V}}(\alpha)= & 2 K L \delta_{0}^{2}\left[\sin ^{2} 2\left(\alpha+\omega_{0}\right)\left\langle\cos ^{2} 2 \Delta_{1}\right\rangle_{\mathrm{av}}\right. \\
& \left.+\cos ^{2} 2\left(\alpha+\omega_{0}\right)\left\langle\sin ^{2} 2 \Delta_{1}\right\rangle_{\mathrm{av}}\right] \\
& \times \int_{0}^{L} \Gamma\left(r_{12}\right)\left[1+A \Psi\left(r_{12}\right)\right] f\left(r_{12}\right) \\
& \times \cos \left[k r_{12} \sin \theta \cos (\alpha-\mu)\right] \mathrm{d} r_{12}
\end{aligned}
$$

where the quantity $A$ is defined by $A=$ $\left\langle\left(\Delta \delta_{1}\right)^{2}\right\rangle_{\text {av }} / \delta_{0}{ }^{2}$ which is associated with the relative magnitude of anisotropy fluctuation.

In case where there are no internal disorders, $\left\langle\left(\Delta \delta_{1}\right)^{2}\right\rangle_{\mathrm{av}}=\left\langle\sin ^{2} 2 \Delta_{1}\right\rangle_{\mathrm{av}}=0,\left\langle\cos ^{2} 2 \Delta_{1}\right\rangle_{\mathrm{av}}=1$, and $f\left(r_{12}\right)=\Psi\left(r_{12}\right)=1$, so that the equation reduces to that for a homogeneous rod with length $L$. On the other hand, with increasing disorders, $\left\langle\cos ^{2} 2 \Delta_{1}\right\rangle_{\mathrm{av}}$ and $\left\langle\sin ^{2} 2 \Delta_{1}\right\rangle_{\mathrm{av}}$ approach to $1 / 2$, and $\Psi\left(r_{12}\right)$ and $f\left(r_{12}\right)$ approach to $\delta\left(r_{12}\right)$, Dirac delta function. For the limiting case, $I_{H_{\mathrm{V}}}(\alpha)$ is a constant and independent of $\alpha, \theta$, and $\mu$, so that the total scattering intensity is also independent of $\theta$ and $\mu$. The scattering reduces to Rayleigh scattering in the limiting case.

If the rod-like texture is composed of mosaic crystal blocks, $f\left(r_{12}\right)$ is shown to be a function of the size of the block and of the orientation disorder from one block to the other, ${ }^{25}$ so that the evaluation of $f\left(r_{12}\right)$ would be of interest. The crystal defects would cause the anisotropy fluctuation.

\section{Results of Numerical Calculations}

Numerical calculations of the theoretical $H_{\mathrm{V}}$ scattering were carried out based upon eq 5 and 18 with the Facom 230-60 Computer, Research Computer Center of Kyoto University. Equation 5 was further modified, prior to calculations, as $I=2 K \int_{0}^{\pi} E(\alpha) E^{*}(\alpha) \mathrm{d} \alpha$, since the integrand is a periodic function with period $\pi$ as seen in eq 18. Moreover, the integration over $r_{12}$ in eq $\mathbf{1 8}$ was analytically carried out prior to the numeri- 
cal calculations in order to reduce the computing time. The calculations were made by giving an arbitrary value for the quantity related to the absolute scattered intensity, since only the relative intensity distributions are of interest in this study.

The effects of the anisotropy and orientation disorders were separately considered. The correlation functions which, in general, decrease asymptotically from unity to zero with increasing distance $\left|r_{12}\right|$ from zero were assumed to be given by the empirical functions,

$$
\Psi\left(r_{12}\right)=\exp \left(-\left|r_{12}\right| / a\right), \quad f\left(r_{12}\right)=\exp \left(-\left|r_{12}\right| / c\right)
$$

where $a$ and $c$ are the "so called" correlation distances.

The two parameters, the correlation distance and the magnitude of the fluctuation, are expected to be interrelated. The interrelating equation derived by Stein and $\mathrm{Chu}^{21}$ was used in this study for the orientation fluctuation.

$$
\begin{aligned}
& \left\langle\cos ^{2} 2 \Delta_{1}\right\rangle_{\mathrm{av}} \\
& =\frac{1}{2}-\frac{1}{16(L / c)^{2}}[1-4(L / c)-\exp (-4 L / c)]
\end{aligned}
$$

Therefore for a large fluctuation with small correlation distance $c$, the values of $\left\langle\cos ^{2} 2 \Delta_{1}\right\rangle_{a v}$ and $\left\langle\sin ^{2} 2 \Delta_{1}\right\rangle_{a v}$ approach to $1 / 2$, the value corresponding to completely random fluctuation. The interrelating equation was not used, however, for the case of anisotropy disorders, so that the values of two parameters were independently given for the numerical calculations.

The results of numerical calculations are shown in Figures 16 to 18 where the $H_{V}$ scattered intensities at $\mu=0$ and $45^{\circ}$ are plotted as a function of the reduced scattering angle $W=$ $(\pi L \sin \theta) / \lambda$ for various disorder parameters and for a special value of $\omega_{0}=45^{\circ}$. The value of $\omega_{0}$ gives rise to $H_{\mathrm{V}}$ scattering patterns with maximum intensities at $\mu=0$ and $(2 n+1) \pi / 2$ and minimum intensities at $\mu=(2 n+1) \pi / 4$ and with maximum angular asymmetry $D_{\mu}$ with respect to $\mu$ defined by $D_{\mu}=I_{H_{\mathrm{V}}}\left(\mu=0^{\circ}\right) / I_{H_{\mathrm{V}}}\left(\mu=45^{\circ}\right)$.

In Figure 16 is shown the effect of the anisotropy fluctuation with various correlation distances specified by $(a / L)$ for a given parameter $A=\left\langle\left(\Delta \delta_{1}\right)^{2}\right\rangle_{\mathrm{av}} / \delta_{0}{ }^{2}=1.0$. The fluctuation generally increases the scattered intensities. The increase in the intensities is greater for smaller values of $(a / L)$, i.e., for greater anisotropy fluctuation. The relative rate of increase of the intensities is greater at $\mu=45^{\circ}$ than at $\mu=0^{\circ}$, and at larger $\theta$ than at smaller $\theta$, indicating, respectively, that (i) the $\mu$-dependence of the intensity (or the

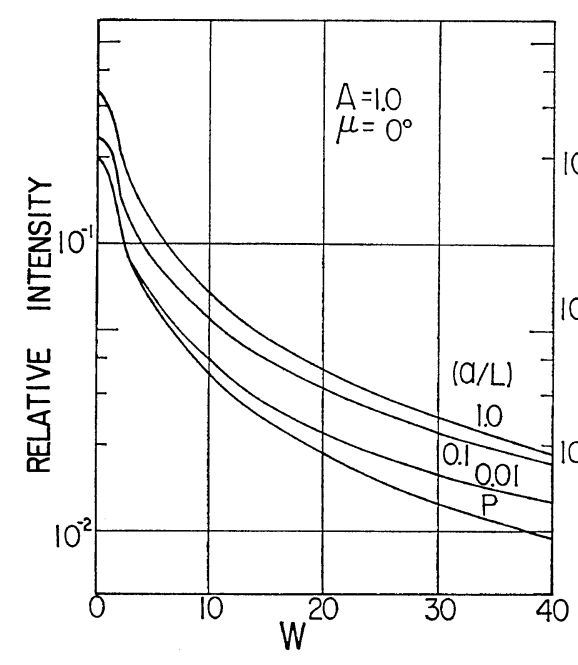

(a)

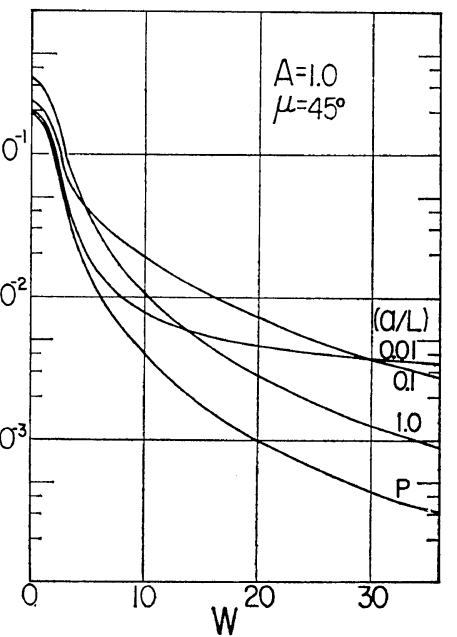

(b)

Figure 16. The effect of the anisotropy disorder on the $H_{\nabla}$ scattering intensities at $\mu=0^{\circ}$ (a) and $45^{\circ}$ (b) as a function of the correlation distance $a$ for $A=\left\langle\left(\Delta \delta_{1}\right)^{2}\right\rangle_{\mathrm{av}} / \delta_{0}^{2}=1.0$. The curve $\mathrm{P}$ corresponds to that of the homogeneous rod. 
Light Scattering from Polymer Films Having a Disordered Anisotropic Rod-Like Texture

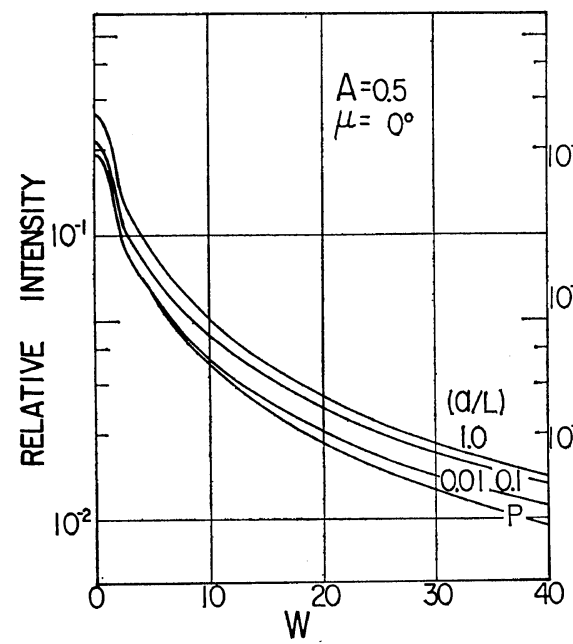

(a)

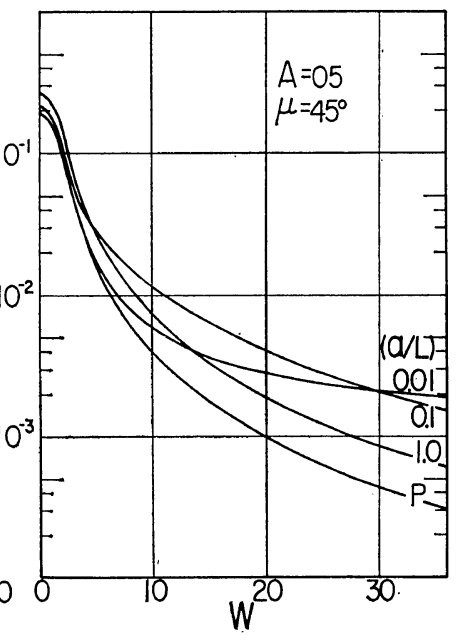

(b)

Figure 17. The effect of the anisotropy disorder on the $H_{\mathrm{V}}$ scattering intensities at $\mu=0^{\circ}$ (a) and $45^{\circ}$ (b) as a function of the correlation distance $a$ for $A=0.5$. The curve $\mathrm{P}$ corresponds to that of the homogeneous rod.

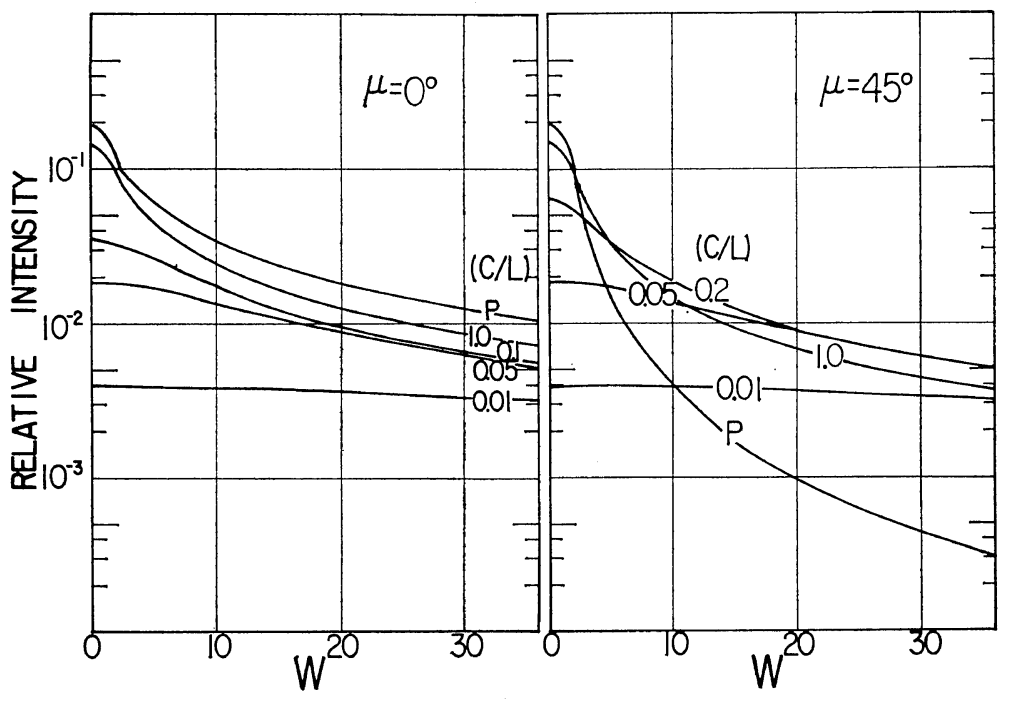

(a)

(b)

Figure 18. The effect of the orientation disorder on the $H_{\mathrm{V}}$ scattering intensities at $\mu=0^{\circ}$ (a) and $45^{\circ}$ (b) as a function of the correlation distance $c$. The curve $\mathbf{P}$ is for the homogeneous rod.

value of $D_{\mu}$ ) decreases with increasing fluctuation and that (ii) the angular dependence of $D_{\mu}$ with respect to $\theta$ is also modified by the fluctuation.

The tendencies may be also seen in Figure 19 where the angular asymmetry $D_{\mu}$ is plotted as a function of $W$ for the same disorder parameters. The values of $D_{\mu}$ continuously increase

Polymer J., Vol. 6, No. 2, 1974 with increasing $W$ for the homogeneous rod. The values of $D_{\mu}$ decrease with increasing disorder, i.e., with decreasing values of $a / L$ for a given value of $W$. The values of $D_{\mu}$ do not continuously increase but reach a maximum value and then decrease with increasing $W$ for large fluctuation, for example, $a / L=0.01$. The 
tendencies qualitatively agree with the experimental results shown in Figure 11.

In Figure 17 is shown the effect of the anisotropy disorder for a smaller value of $\left\langle\left(\Delta \delta_{1}\right)^{2}\right\rangle_{\mathrm{av}} /{\delta_{0}}^{2}$ $(=0.5)$, the relative magnitude of disorders. The results are similar to those for $\left\langle\left(\Delta \delta_{1}\right)^{2}\right\rangle_{\mathrm{av}} / \delta_{0}{ }^{2}=1.0$ except for the fact that the effect is smaller, for the smaller value of $\left\langle\left(\Delta \delta_{1}\right)^{2}\right\rangle_{\mathrm{av}} / \delta_{0}{ }^{2}$, i.e., the decrease of $D_{\mu}$ for a given values of $a / L$ is smaller for the smaller value of $\left\langle\left(\Delta \delta_{1}\right)^{2}\right\rangle_{\mathrm{av}} / \delta_{0}{ }^{2}$ than for the larger value. It should be noted that the disorder decreases also the angular asymmetry with respect to $\theta$ as can be clearly seen in Figures 16 and 17.

In Figure 18 is shown the effect of the orientation disorders on the $H_{\mathrm{V}}$ scattering intensities at $\mu=0$ and $45^{\circ}$ for $\omega_{0}=45^{\circ}$. The orientation disorder also decreases the angular asymmetry with respect to $\theta$ and $\mu$, similar to the anisotropy disorder. In contrast to the anisotropy disorder, the orientation disorder, however, decreases the intensity at $\mu=0^{\circ}$ and increases the intensity at $\mu=45^{\circ}$ and at large $\theta$ with increasing the disorder, so that the effects of the disorder are much greater than the effects of the anisotropy disorder. In Figure 19 are shown the effects of the orientation disorden on $D_{\mu}$. The tendencies are similar to those of the anisotropy disorder, except for the fact that the maximum in $D_{\mu}$ is not observed for the orientation disorders.

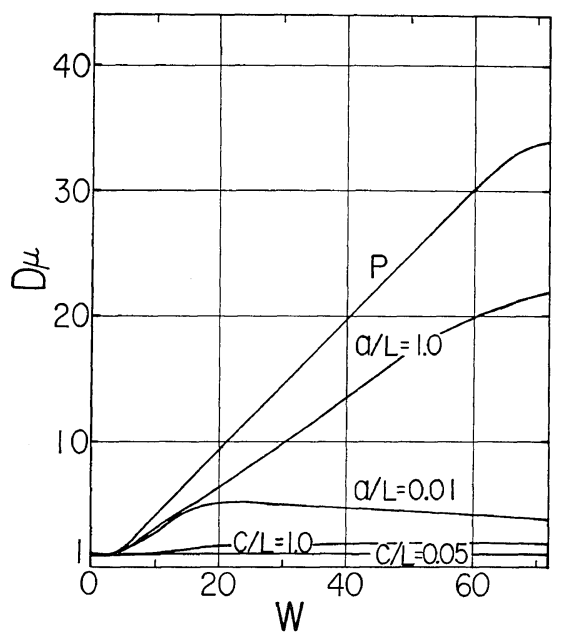

Figure 19. The angular asymmetry $D_{\mu}$ for the orientation and anisotropy disorders. $\omega_{0}=45^{\circ}$. The curve $\mathbf{P}$ is for the homogeneous rod.
The arithmetric averages of the intensities at $\mu=0$ and $45^{\circ}$ are independent of the orientation of the optical axes even for the disordered rods as expected from eq 18. Their angular dependences with respect to $W$ or $\theta$ decrease remarkably with increasing anisotropy and orientation disorders.

\section{DISCUSSION}

As shown in Figure 20 the experimental values of $D_{\mu}$ are small compared with those of the homogeneous rods, and do not increase, with increasing $\theta$, to the extent as expected for the homogeneous rod. The experimental values of $D_{\mu}$ level off at large scattering angles as for the collagen $\mathrm{C}-2$ specimens or decrease toward unity with increasing scattering angles as for the collagen $\mathrm{C}-1, \mathrm{C}-3$ and PTFE- $\mathrm{L}$ and $-\mathrm{H}$ specimens, the tendencies of which are difficult to explain by the homogeneous rod theory or by the polydispersity of the rod in terms of the size but can be explained, at least qualitatively, by introducing the disorders in the rods.

It should be noted that the disordered rod theory, however, has difficulty in accounting for the large decrease of $D_{\mu}$ observed with increasing angles as for the C-1, C-3 and PTFE-L films, unless large disorders, especially large anisotropy disorder, are involved in the system, or unless the two types of disorder are coupled in a very

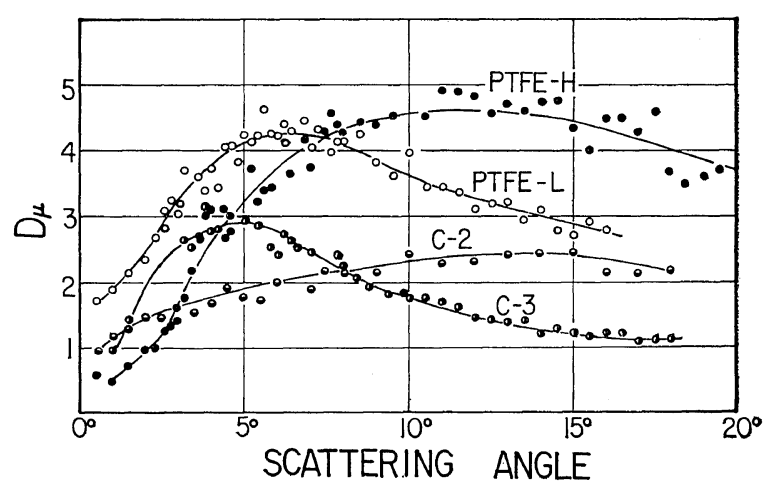

Figure 20. Experimental angular asymmetry $D_{\mu}$ for the PTFE-L, PTFE-H C-2, and C-3 specimens. For the PTFE-L specimens, $D_{\mu}$ is defined as $I_{H_{\mathrm{V}}}\left(\mu=45^{\circ}\right) / I_{H_{\mathrm{V}}}\left(\mu=0^{\circ}\right)$ instead of $I_{H_{\mathrm{V}}}\left(\mu=0^{\circ}\right) /$ $I_{H_{\mathrm{V}}}\left(\mu=45^{\circ}\right)$ for the PTFE-H, C-2, and C-3 specimens. 


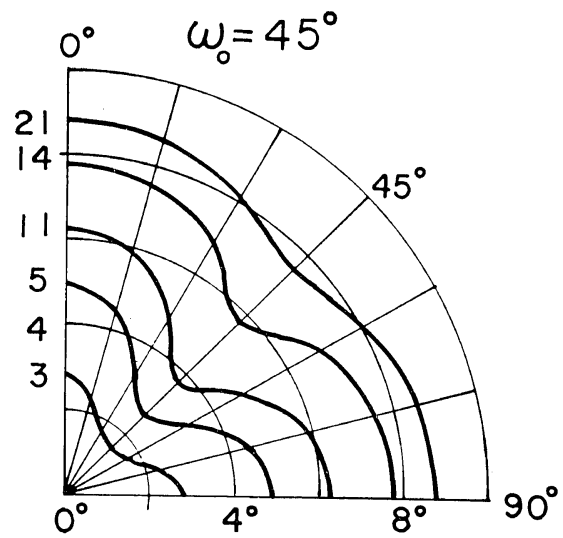

(a)

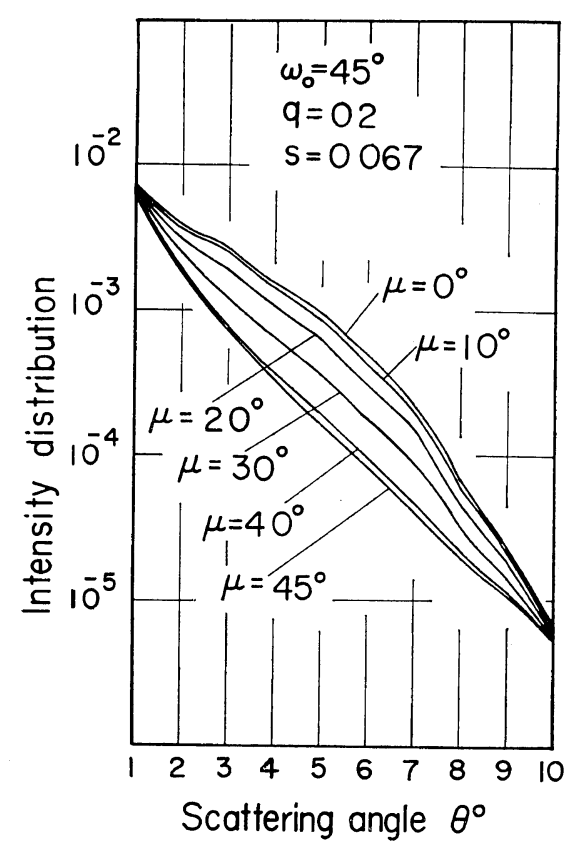

(b)

Figure 21. The calculated $H_{\mathrm{V}}$ scattering patterns (a) and intensity distributions (b) for the anisotropic rectangular sheets in a two-dimensional plane normal to $s_{0} ;(L / \lambda)=30, D / L=0.2$ and $G / L=0.067$.

specific manner. It is possible that there are other factors which, alone or together with the disorders, account for the difficulty. In fact it turned out that the value of $D_{\mu}$ decreases sensitively at large $\theta$ when the effect of finite lateral dimensions of the rod are introduced in the theory as will be reported in a subsequent paper. ${ }^{6}$
In Figure 21 is shown an example of the effect of finite lateral dimensions of the rods on the $H_{\mathrm{V}}$ scattering patterns (a) and intensity distributions (b) both for $\omega_{0}=45^{\circ}$. The rod-like textures are assumed to be rectangular plates in shape and to be oriented randomly in a two-dimensional plane. The plates have dimensions of length $L$ and width $D$ parallel to the plane and thickness $G$ perpendicular to the plane. The optical axes are again constrained in the plane and make an angle $\omega_{0}$ within the sheet with respect to a direction parallel to the edge of length $L$.

As seen in Figure 21a, the $\mu$-dependences increase and then decrease with increasing, $\theta$, a tendency which is in good agreement with the experimental contour pattern as shown in Figure 12a. The tendency is also seen clearly in the calculated intensity distributions shown in Figure 21b. When the width $D$ is decreased, the appearances of the calculated $H_{\mathrm{V}}$ patterns approach to those of Figure 12b.

The discussion so far was based on a special value of $\omega_{0}=45^{\circ}$. The effects of $\omega_{0}$ on the angular distribution of the intensities with respect to $\theta$ and $\mu$ can be analyzed from eq 18 . It follows, from the equation, that

$$
\begin{aligned}
{\left[I_{H_{\mathrm{V}}}(\mu=\right.} & \left.\left.0^{\circ}\right)+I_{H_{\mathrm{V}}}\left(\mu=45^{\circ}\right)\right] / 2 \\
= & K L{\delta_{0}}^{2} \int_{t=0}^{\pi} \int_{r=0}^{L} \Gamma(r)[1+A \psi(r)] f(r) \\
& \times \cos (k r \sin \theta \cos t) \mathrm{d} r \mathrm{~d} t
\end{aligned}
$$

and

$$
\begin{aligned}
{\left[I_{H_{\mathrm{V}}}(\mu=\right.} & \left.\left.0^{\circ}\right)-I_{H_{\mathrm{V}}}\left(\mu=45^{\circ}\right)\right] / 2 \\
= & -K L \delta_{0}{ }^{2} \cos 4 \omega_{0}\left[2\left\langle\cos ^{2} 2 \Delta_{1}\right\rangle_{\mathrm{av}}-1\right] \\
& \times \int_{t=0}^{\pi} \cos 4 t \int_{r=0}^{L} \Gamma(r)[1+A \psi(r)] f(r) \\
& \times \cos (k r \sin \theta \cos t) \mathrm{d} r \mathrm{~d} t
\end{aligned}
$$

Hence the value of $\omega_{0}$ does not alter the angular dependence of the arithmetric average of eq 21 with respect to $\theta$. The $\theta$-dependence varies only with the disorders. However the angular dependences of the intensities with respect to $\mu$, depend very much on the value of $\omega_{0}$ as shown in eq 22 , so that the value of $D_{\mu}$ is also affected by $\omega_{0}$.

Acknowledgments. Part of this work was supported by a grant from the Scientific Research 
Funds (Kagaku Kenkyu-hi, 743020-1973) of the Ministry of Education, Japan, and a grant from the Japan Synthetic Rubber Co. Ltd., Tokyo, Japan.

The authors wish to express their gratitude for the assistance of the Research Laboratory, Japan Leather Co. Ltd., Tokyo, Japan, where the test films of collagen were prepared. The authors are also indebted to the Research Laboratory, Daikin Industries, Osaka, Japan, for their kind assistance in the preparation of the PTFE films and the scanning electron micrographs.

\section{REFERENCES}

1. M. B. Rhodes and R. S. Stein, J. Polym. Sci., Part A-2, 7, 1539 (1969).

2. M. Moritani, N. Hayashi, A. Utsuo, and $H$. Kawai, Polymer J., 2, 74 (1971).

3. Y. Murakami, N. Hayashi, T. Hashimoto, and H. Kawai, ibid., 4, 452 (1973).

4. J. J. van Aartsen, Eur. Polym. J., 6, 1095 (1970).

5. N. Hayashi and H. Kawai, Polymer J., 3, 140 (1972).

6. M. Matsuo, S. Nomura, T. Hashimoto, and H. Kawai, Polymer J., 6, 151 (1974).

7. M. B. Rhodes and R.S. Stein, J. Appl. Phys., 39, 4903 (1968).

8. T. Hashimoto, Y. Murakami, Y. Okamori, and H. Kawai, paper to be submitted to Polymer $J$.

9. G. C. Adams and R. S. Stein, J. Polym. Sci., Part A-2, 6, 31 (1968).

10. N. Hayashi, Y. Murakami, M. Moritani, T.
Hashimoto, and H. Kawai, Polymer J., 4, 560 (1973).

11. J. Borch and R. H. Marchessault, J. Polym. Sci., Part C, 28, 153 (1969).

12. R. J. Samuels, ibid., Part A-2, 7, 1197 (1969).

13. J.C.W. Chien and E. P. Chang, Macromolecules, 5, 610 (1972).

14. W. Chu, paper to be submitted.

15. K. Nagatoshi, T. Hashimoto, and H. Kawai, paper to be submitted.

16. R. S. Stein and M. B. Rhodes, J. Appl. Phys., 31, 1873 (1960).

17. C. W. Bunn, A. J. Cobbold, and R. P. Palmer, J. Polym. Sci., 28, 356 (1958).

18. C. J. Speerschneider and C. H. Li, J. Appl. Phys., 33, 1871 (1962).

19. N. K. J. Symons, J. Polym. Sci., Part A, 1, 2843 (1963).

20. A. E. M. Keijizers, J. J. van Aartsen, and W. Prins, J. Amer. Chem. Soc., 90, 3167 (1968).

21. R. S. Stein and W. Chu, J. Polym. Sci., Part A-2, 8, 1137 (1970).

22. T. Hashimoto and R. S. Stein, ibid., Part A-2, 9, 1747 (1971).

23. R. S. Stein and T. Hashimoto, ibid., Part A-2, 9, 517 (1971).

24. T. Hashimoto, R. P. Prud'homme, D. A. Keedy, and R. S. Stein, J. Polym. Sci., Polym. Phys., Ed., 11, 693 (1973).

25. R. S. Stein and S. N. Stidham, J. Appl. Phys., 35, 42 (1964).

26. See for example, A. Guinier and G. Fournet, "Small-Angle Scattering of X-Rays", John Wiley \& Sons, Inc., London, Chapmann and Hall, Ltd., 1955. 